\title{
Fostering Distributed Business Logic in Open Collaborative Networks: an integrated approach based on semantic and swarm coordination
}

Francesco P. Appio ${ }^{1}$, Mario G. C. A. Cimino ${ }^{2, *}$, Alessandro Lazzeri ${ }^{2}$, Antonella Martini ${ }^{3}$, Gigliola Vaglini $^{2}$

${ }^{1}$ Pôle Universitaire Léonard de Vinci, Research Center (Business Group)

12 av. Léonard de Vinci, 92916 Paris La Défense Cedex, France

e-mail: francesco.appio@ devinci.fr

${ }^{2}$ Department of Information Engineering

University of Pisa

Largo Lucio Lazzarino 1 - 56122 Pisa, Italy

e-mail: mario.cimino@unipi.it; alessandro.lazzeri@for.unipi.it; gigliola.vaglini@unipi.it

\section{${ }^{3}$ Department of Energy, System, Territory and Construction Engineering}

University of Pisa

Largo Lucio Lazzarino 1 - 56122 Pisa, Italy

e-mail: a.martini@ing.unipi.it

* Corresponding author

Mario G. C. A. Cimino

E-mail: mario.cimino@unipi.it

Phone: +39050 2217455

Fax: +390502217600

\section{Abstract}

Given the great opportunities provided by Open Collaborative Networks (OCNs), their success depends on the effective integration of composite business logic at all stages. However, a dilemma between cooperation and competition is often found in environments where the access to business knowledge can provide absolute advantages over the competition. Indeed, although it is apparent that business logic should be automated for an effective integration, chain participants at all segments are often highly protective of their own knowledge. In this paper, we propose a solution to this problem by outlining a novel approach with a supporting architectural view. In our approach, business rules are modeled via semantic web and their execution is coordinated by a workflow model. Each company's rule can be kept as private, and the business rules can be combined together to achieve goals with defined interdependencies and responsibilities in the workflow. The use of a workflow model allows assembling business facts together while protecting data source. We propose a privacy-preserving perturbation technique which is based on 
digital stigmergy. Stigmergy is a processing schema based on the principle of self-aggregation of marks produced by data. Stigmergy allows protecting data privacy, because only marks are involved in aggregation, in place of actual data values, without explicit data modeling. This paper discusses the proposed approach and examines its characteristics through actual scenarios.

Keywords: open collaborative network; workflow; business rule; web ontology; data perturbation; stigmergy.

\section{Introduction and Motivation}

\subsection{Moving towards Open Collaborative Networks}

A progressive opening of the boundaries of the companies is increasingly taking place. Companies started applying this philosophy since the 1990s, by looking at the enormous potential outside their walls, even those of their supply chains. In such a context, borders are constantly blurring, formal and informal networks interplay, companies have multiple memberships to dynamic and evolving structures.

From an historical perspective, three decades have shaped the environmental conditions for enabling inter-enterprise collaboration (e.g., Camarinha-Matos, 2013; Curley and Salmelin, 2013, Gastaldi et al., 2015). The 1990s were characterized by a competitive landscape leveraging inward-looking systems, concentrated on making enterprise more efficient in isolation, where collaboration activities were mainly focused on signing agreements with supply chain partners. In such context, where the Internet was still in infancy, the debate about the role of information technology in future manufacturing systems was still ongoing, and organizations were trying to structure policies and mechanisms to become more specialized and inter-connected (Browne et al., 1995). Some firms began to employ the early concepts of Extended Enterprise (EE), i.e., the principle that a dominant enterprise extends its boundaries to all or some of its suppliers. More simply, the early concept of EE meant placing the manufacturing systems in the context of the value chain (Porter, 1985). Such extended configurations lead to Computer Integrated Manufacturing (CIM) systems. Indeed, from one side the challenge of CIM was to realize integration within the factory, from the other side the challenge to manufacturing was shifting to facilitate inter-enterprise networking across the value chain. In the late 90 s, concepts such as Virtual Enterprises (VEs) and Virtual Organizations (VOs) started diffusing, although still at the level of single - and rather isolated - networks. More precisely, VEs represent dynamic and often short-term alliances of enterprises that come together to share skills or core competencies and resources, in order to better respond to business opportunities, and whose cooperation is supported by computer networks ( $\mathrm{Li}$ et al., 2014). An EE can be seen as a particular case of a VE. VOs generalize the concept of VEs, because it is not limited to an alliance for profit, but to achieve missions/goals (Camarinha-Matos and Afsarmanesh, 2007).

The 2000s were characterized by ICT advancements enabling new collaborative partnerships modes and the concept of Collaborative Networked Organization (CNO), which further generalizes VO. A CNO is an organization whose activities, roles, governance rules, are 
manifested by a network consisting of a variety of entities (e.g., organizations and people). Such entities are largely autonomous, geographically distributed, and heterogeneous in terms of their operating environment, culture, social capital and goals. But they collaborate to better achieve common or compatible goals, thus jointly generating value, and whose interactions are supported by computer network. Since not all forms of collaborative partnership imply a kind of organization of activities, roles, and governance rules, the concept of Collaborative Network $(\mathrm{CN})$ further generalize the collaborative partnership (Camarinha-Matos and Afsarmanesh, 2007; CamarinhaMatos et al., 2009; Romero and Molina, 2010). In the meanwhile, a progressive opening of the companies boundaries enabled what has been defined the Open Innovation paradigm (Chesbrough, 2003, Appio et al., 2016), in which externally focused, collaborative innovation practices were adopted.

A deep mutation has been occurring in the last decade, the 2010s, in which the competitive landscape morphed with the introduction of the Ecosystems perspective (Baldwin and Von Hippel, 2011; Curley and Samlelin, 2013). A new paradigm has been opening up, stressing the salient characteristics of the variety of CNs discussed by Camarinha-Matos et al. (2009). We label it as Open CNs (OCNs). OCNs are based on principles of integrated collaboration, co-created shared value, cultivated innovation ecosystems, unleashed exponential technologies, and extraordinarily rapid adoption (Curley and Salmelin, 2013). They also capture the elemental characteristics of the constant transformation of networks ecosystems: continual realignment of synergistic relationships of people, knowledge and resources for both incremental and transformational value co-creation (Ramaswamy and Gouillart, 2010). Through relationships, value co-creation networks evolve from mutually beneficial relationships between people, companies and investment organizations. A continual realignment of synergistic relationships of people, knowledge and resources is required for vitality of the ecosystem. Requirements for responsiveness to changing internal and external forces make co-creation an essential force in a dynamic innovation ecosystem (Russell et al., 2011). In the third era, borders are further blurring, formal and informal networks interplay, companies have multiple memberships to dynamic and evolving structures. In OCNs contexts where ubiquity is for the first time allowed, the probability of break-away improvements increases as a function of diverse multidisciplinary experimentation, a controlled process, addressing systematically a set of steps, supported by different mechanisms and approaches to characterize the management functionalities of a $\mathrm{CN}$ during its entire lifecycle.

In the next section we introduce the distinctive characteristics of the OCNs, trying to disentangle the needs along with the challenges.

\subsection{Characterizing Open Collaborative Networks (OCNs)}

Camarinha-Matos and Afsarmanesh $(2005,2009)$ provide a comprehensive characterization of the $\mathrm{CN}$, defining it as a network consisting of a variety of entities (e.g. organizations and people) that are largely autonomous, geographically distributed, and heterogeneous in terms of their operating environment, culture, social capital and goals, but that collaborate to better achieve common or compatible goals, thus jointly generating value, and whose interactions are supported by computer network. Moving from this definition, we want to characterize a type of $\mathrm{CN}$ in which 
more unstructured and self-organizing behaviors can be considered (e.g., Panchal 2010; Levine and Prietula, 2013; Baldwin and Von Hippel, 2011; Bonabeau et al., 1997; Holland et al., 1999). For this purpose, this section aims at characterizing the OCN according to the key dimensions.

An OCN can be thought of as entailing all the characteristics of a $\mathrm{CN}$ but is different under the following respects:

1. it allows agents to take advantage of signals echoing the three layers (Moore, 1996) namely, business ecosystem (trade associations, investors, government agencies and other regulatory bodies, competing organizations that have shared product $\&$ service attributes, business processes and organizational arrangements, other stakeholders, labor unions), extended enterprise (i.e. direct customers, customers of my customers, standard bodies, suppliers of complementary products, suppliers of my suppliers), and core business (core contributors, distribution channels, direct suppliers);

2. it is inspired by ecosystem perspective, and then deals with a variety of structures ranging from communities, to very loosely coupled agents coexisting and influencing each other. The ecosystem, in its structural and functional openness, is the fertile ground for more complex networks to grow and interact (Iansiti and Levien, 2004);

3. it subsumes that agents self-organize into more or less structured networks maximizing the returns on the inside-out/outside-in practices (or knowledge inflows and outflows); the ecosystem perspective potentially allows for a simultaneous reduction of both error types by decreasing the risk of information overload, improving the ability to handle complexity and minimizing interpretation biases (Velu et al., 2010). About the two errors, a type I interpretation error (false positive) consists in detecting a specific market trend when there is actually none. Noise is just wrongly interpreted as a valuable signal of an important development in customer needs, competitor behavior or technological progress. Conversely, a type II interpretation error (false negative) consists in failing to observe an important market trend, when in truth there is one. Meaningful market signals are thus overlooked or wrongly interpreted as meaningless. Firms operating in (closed) CNs have to trade-off those type I and type II errors, both of which can be extremely costly;

4. it is less hierarchical and more oriented towards self-organization (Steiner et al., 2014; Panchal, 2010; Jelasity et al., 2006). Self-organization is the process in which pattern at the global level of a system emerges solely from numerous interactions among the lowerlevel components of the system. Moreover the rules specifying the interactions among the system's component are executed using only local information, without reference to the global pattern. Self-organization relies on four ingredients: a) positive feedback, b) negative feedback, c) amplification of fluctuations, and d) multiple interactions. The behavior of entities may be attributed to physical behavior in the case of physical entities and decisions in the case of human participants. The behaviors of entities are based on local information available to them, which changes as the entities interact with each other. These changes in local information may result in positive or negative feedback; a balance between these two types of feedback results in self-organizing behavior; 
5. it tolerates (and balances) two different types information exchange: direct and indirect. Direct interactions involve direct information exchange between different individuals, which changes their local information, and hence, their decisions. In the case of indirect interactions, the individual actions affect the environment and modify it. Such indirect interaction of entities with the environment plays an important role in achieving coordination through self-organization mechanisms (Kiemen, 2011).

Overall, OCNs inherit all the fundamental characteristics of the CNs, while the attribute Open describe something more (Table 1):

Table 1. A comparative analysis of CNs and OCNs.

$\begin{array}{ccc}\text { Characteristics } & \text { Collaborative } & \text { Open Collaborative } \\ & \text { Networks (CNs) } & \text { Networks (OCNs) }\end{array}$

\begin{tabular}{lcc}
\hline Variety of agents & + & ++ \\
\hline Autonomy of agents & + & ++ \\
\hline Geographical distribution & + & + \\
\hline Heterogeneity of agents & + & ++ \\
\hline Working on common goals & ++ & + \\
\hline Support of ICT networks & + & +
\end{tabular}

Ecosystem perspective ++

Structured interactions $\quad++\quad+$

Addressing interpretation errors (Type I-II) $\quad+\quad++$

Variety of collaboration modes $++\quad++$

Self-organization practices ++

Direct communications $\quad++\quad+$

Indirect communications ++

166 + moderate intensity of the characteristic; ++ high intensity of the characteristic which rapid and fluid configuration of $\mathrm{CNs}$ may arise, once recognized business opportunities to exploit (Afsarmanesh and Camarinha-Matos, 2005); on the other side, they imply that criteria,

171 metrics, and assessment are likely to become even more influential as evaluations move online,

172 becoming widespread, consumer based, globally dispersed, and widely accessible (Orlikowski and

173 Scott, 2013). Figure 1 extends the network configurations advanced by Camarinha-Matos and

174 Afsarmanesh (2009) in a way that all the described dimensions are taken into account: 


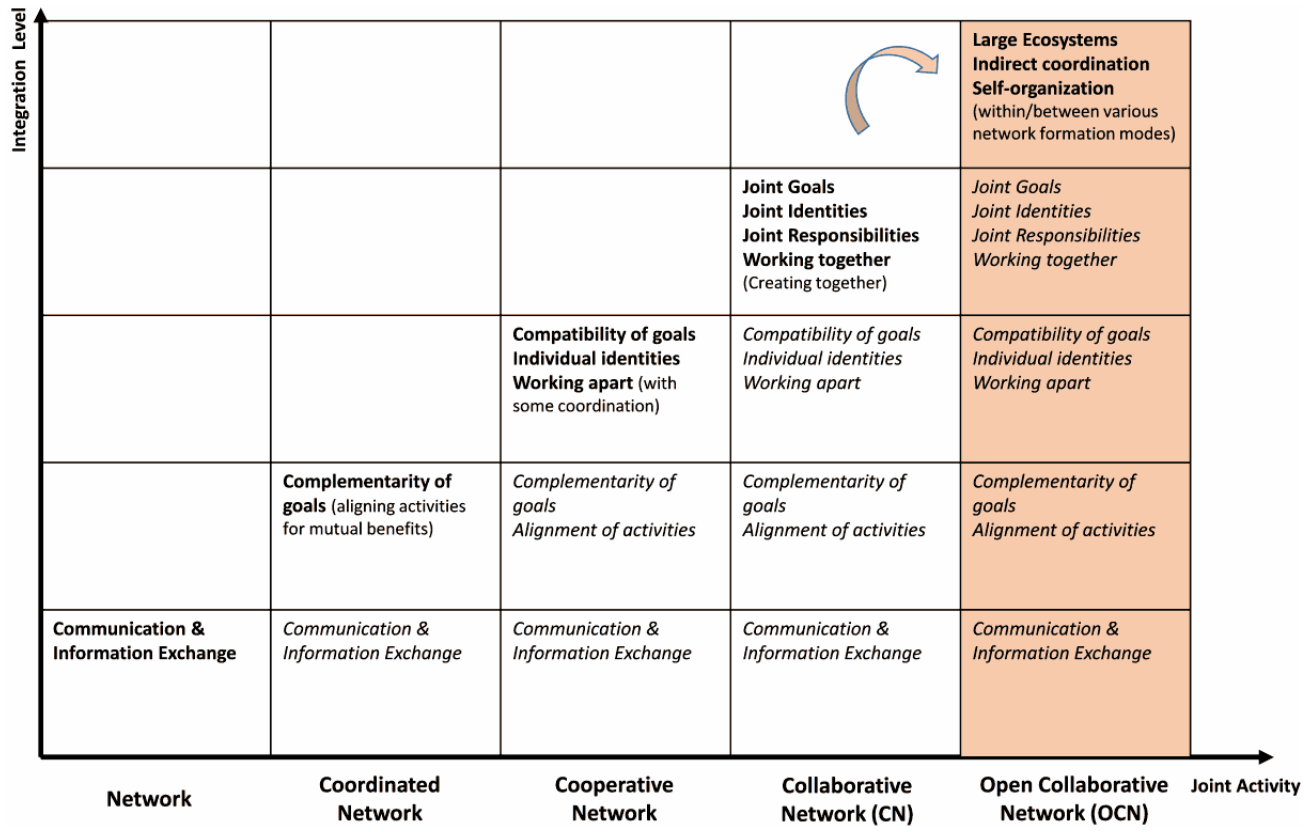

176 Figure 1. Evolution from Network to Open Collaborative Network (adapted from Camarinha-

177 Matos and Afsarmanesh, 2009).

The aim of this paper is then threefold: first, we introduce a novel concept which represents an important evolution with respect to the existing characterization of $\mathrm{CNs}$; second, and strictly related to the introduction of this new concept, a novel approach to distributed business logic is developed in order to make this concept working, bringing together methods which - to the best of our knowledge - lack sound investigations in the current literature; third, a system architecture to support the proposed approach has been designed, developed, and experimented. In the literature the benefits of collaboration are clear, but it is also apparent that different paths to a successful collaboration can be envisaged, since many drivers exist and new ones tend to appear. The novel capabilities of the proposed system reside in keeping enterprises prepared to manage different kinds of business collaborations, entailing support for abstraction and advanced modeling techniques in combination.

What follows in Section 2 better contextualizes OCNs by providing the reader with the underlying business requirements. Section 3 shows how - and to what extent - technology can make the business requirements working in an integrated fashion; then, the integrated system is introduced. Sections 4 and 5 will introduce the building blocks of the system against a pilot study. Section 6 describes: (i) how to integrate all the building blocks in a system architecture, (ii) how the system can be administered, and (iii) how it has been experimented. Section 7 discusses the main findings and opens to potential future research avenues.

\section{Business requirements for Open Collaborative Networks}

The key characteristics that basically distinguish OCNs from previous contexts are the 
interactions and complex exchange dynamics (e.g., Panchal 2010; Levine and Prietula, 2013; Baldwin and Von Hippel, 2011). These characteristics result in self-organization of participants, activities, and organizational (community) structures, as opposed to hierarchical structures in traditional product development (Bonabeau et al., 1997; Holland et al., 1999). Self-organization means that a functional structure appears and maintains spontaneously. The control needed to achieve this must be distributed over all participating components. Overall, OCNs can be thought of as distributed systems which are different from centralized and decentralized ones (Dhakal, 2009; Andrés and Poler, 2013; Andrés and Poler, 2014). Indeed, in distributed systems all agents are networked on the basis of equality, independence, and cooperation. The greatest advantage of distribution is that the resilience of the system increases with the increase in the number of participants. Nowadays, distributed systems can be made possible thanks to the advancements in the ICT infrastructures. Distributed systems are also known as layer-less system or hierarchy-less system in that they use lateral (horizontal) protocols based on equality of relationship as opposed to a decentralized system (also known as layered system or hierarchical system), which uses hierarchical protocols where a higher agent must always control the lower ones. Both centralized and decentralized systems thrive on the use of authority, something which is really smoothed in the cases of OCNs. In the literature, Andrés and Poler (2013) identify and analyze strategic, tactical, and operational issues arising in collaborative networks, proposing a classification matrix for the most relevant ones. In a more recent study, they also identify relevant collaborative processes that non-hierarchical manufacturing networks perform (Andrés and Poler, 2014). A novel approach supporting unstructured networked organization is presented in (Loss and Crave, 2011). Here, the authors explore the concept of agile business models for CNs, describing a theoretical framework. Ollus et al. (2011) presented a study aimed to support the management of projects in networked and distributed environments. Collaborative management includes shared project management, which means delegation of management responsibility and some extent of self-organization. The management may in many cases be non-hierarchical and participative with

The general objectives of a OCNs (e.g., Brambilla et al., 2011a, 2011b; Msanjila and Afsarmanesh, 2006; Msanjila and Afsarmanesh, 2011; Romero et al., 2009; Romero and Molina, 2011) can be then articulated into different requirements: (i) transparency: to make the execution of shared procedures more visible to the affected stakeholders; (ii) trust: to deploy measurable elements that can establish a judgment about a given trust requirement; (iii) participation: to engage a broader community to raise the awareness about, or the acceptance of, the process outcome; (iv) activity distribution: to assign an activity to a broader set of performers or to find appropriate contributors for its execution; (v) decision distribution: to separate and distribute decision rules that contribute to the taking a decision; (vi) social feedback: to acquire feedback from stakeholders along the work-flow, for process improvement; (vii) knowledge and information sharing: to disseminate knowledge and information in order to improve task execution without market disruption; (viii) collaboration readiness: to grasp partners' preparedness, promptness, aptitude and willingness; (ix) enabling ICT: to support collaborative activities in OCNs. Overall, an extended perspective on characterizing the collaborative capability (Ulbrich et al., 2011) and 
how to make it work through appropriate governance mechanisms are needed (Clauss and Spiety, 2015; Heindenreich et al., 2014).

It follows a more detailed explanation of how - and to what extent - it is possible to identify patterns and technologies supporting OCNs business requirements. In Section 3, business requirements will be better focused on a technological view.

\subsection{Managing knowledge via workflow technology}

In OCNs contexts if, on one side, firms must develop the ability to recognize the value of new external knowledge, on the other side, they have to assimilate and utilize it for commercial ends and they have to integrate it with knowledge that has been generated internally. They must develop absorptive capacity (Fabrizio, 2009) depending on their knowledge integration and generation mechanisms, many of which embedded in its products, processes and people (Escribano et al., 2009). This process of acquiring and internally using external knowledge has been labelled “inbound open innovation” (Chesbrough, 2003). Empirical studies have consistently found that firms perform more inbound than outbound activities (e.g., Chesbrough and Crowther, 2006), this openness usually taking the form of a heightened demand for external knowledge and other external inputs in the innovation process (Fagerberg, 2005); however, firms still fail to capture its potential benefits (Van de Vrande et al. 2009). Indeed, past studies (e.g., Deeds and Hill, 1996; Katila and Ahuja, 2002; Rothaermel and Deeds, 2006) have found that the process of external search can be ineffective over a certain effort due to firm's bounded rationality and limited information processing. Since the late 1980s, workflow technology (i.e. workflow modeling and workflow execution (Leymann and Roller, 2000)) has been used to compose higher-level business functionality out of individual (composed or non-composed) functions. Such technologies have today the potential to provide solutions for the effective management of knowledge inflows. Workflow-based coordination as a system for tasks routing and allocation, can be thought of as the first place where knowledge is created, shared and used (Reijers et al. 2009).

\subsection{Adopting and using metrics and indicators}

With the explosion of diverse types of information in OCNs in general, and in OCNs in particular, analytics technologies that mine structured and unstructured data to derive insights are now receiving unprecedented attention (Davenport and Harris, 2007; Prahalad and Krishnan, 2008). Today's analytics must be operated firms wide, deep, and at a strategic level (Davenport et al., 2010). A wide range of unstructured data from firms' internal as well as external sources is available (Chen et al., 2011), enabling a broader set of industry partners to participate. In OCNs, under this model, all entities collaborate and co-develop high value analytics solutions. Well (2009) properly frames them under the label "collaborative analytics" namely, a set of analytic processes where the agents work jointly and cooperatively to achieve shared or intersecting goals. They include data sharing, collective analysis and coordinated decisions and actions. Collaborative analytics, while encompass the goals of their conventional counterparts, seek also to increase 

entire business (Well, 2009; Chen et al., 2012).

\subsection{Ontologies and decision rules}

283 Fundamental to collaborative efforts in OCNs is what Jung (2011) defines as "contextual synchronization", facilitating the mutual understanding among the members (Afsarmanesh and Ermilova, 2007; Plisson, 2007; Romero et al., 2007, 2008), agents should at least define which ontologies rule collaborative efforts. While Jung (2011) considers online communities of individual users, we are trying to adopt an organizational point of view in that the OCN is populated with organizational agents. Common and flexible ontology establishment goes through a set of management activities and supporting tools for OCNs ontology adaptation into a specific OCN domain sector, for OCN ontology evolution during the OCN lifecycle, as well as for OCN ontology learning process (Ermilova and Afsarmanesh, 2006; Plisson, 2007; Chen, 2008). The evolutionary trait of ontologies should be considered due to the high speed in which collaboration in OCNs may expire; to this end, e.g. an Ontology Library Systems (OLS) in more than necessary (Simões et al., 2007).

Overall, in OCNs, ontologies may help under several respects (Zelewski, 2001; Bullinger, 2008): (i) to overcome language barriers among participating members: different language and knowledge cultures rules can be captured and 'translated' by an ontology; (ii) to allow the internal integration of information systems which are today both technically driven and governed by managerial or customer oriented understanding; (iii) to enable semantic access to the knowledge in OCNs; (iv) to coordinate collaborative actors with different knowledge backgrounds. This can lead to a number of potential applications, e.g. the integration of information and of systems for computer-supported cooperative work (CSCW) between companies of the same or of different domains.

\subsection{Information sharing policies}

Information reduces uncertainty in OCNs (Fiala, 2005) and aids in integrating flows and functions across working groups such as partners (e.g., Barut et al., 2002; Krovi et al., 2003; Patnayakuni et al., 2006). This reduction of uncertainty is useful as it saves organizational time and cost by minimizing alternate decisions that arise due to uncertainty (Durugbo, 2015). Furthermore, the flow of information is important for managing interactions and negotiations during collaboration activities and for combining the work of individual agents. Agents exchanging information in OCNs should confront with two characteristic: 1) trails, in order to identify new business opportunities and organizations to partner with; trails vanish over time realizing temporal evolution dynamics of OCNs; 2) information perturbation, as enabler of collaboration as privacy and unveiling sensitive information of highly competitive value; our context may be assimilated to the partial-information problem formulated by Palley and Kremer (2014), in which the agent only learns the rank of the current option relative to the options that have already been observed. It is clear that information is something which is capable of having a 
protect the economic value of information, it can be provided by using a privacy-preserving mechanism.

\subsection{Governance requirements}

2.6 A number of approaches about OCNs governance may be adopted and adapted; however, almost all the existing ones are devoted to classical networks which are static in nature (Rabelo et al., 2014).. Some of them underlie the importance of at least three types of governance: transactional governance, relational governance, institutionalized governance (Clauss and Spieth, 2015). Transactional governance studies have focused on the deployment of rules and contracts to safeguard transactions from opportunistic behavior (Puranam and Vanneste, 2009). These are specified in order to formalize processes, activities and roles, define responsibilities and justify consequences in case of disputes. On the other hand, studies concerned with relational governance emphasizing inherent and moral control, governing exchanges through consistent goals and cooperative atmospheres. Trust has been emphasized as a fundamental element of relational governance (Das and Teng, 1998). It has an even greater effect if relational norms between partners establish consistent role behaviours that are in line with partners' joint interests (Tangpong et al., 2010). Institutionalized governance covers a separate functional unit responsible for an active network management (Heidenreich et al., 2014). OCN orchestration mentions activities that enable and facilitate the coordination of the network and the realization of the innovation outputs (Ritala et al., 2009). The orchestrator is responsible for discretely influencing other firms and to support the appropriate conditions for knowledge exchange and innovation. However, being the OCN potentially a highly un-structured $\mathrm{CN}$, the aforementioned forms of governance may be thought of as emergent (Wang et al., 2011).

\section{Establishing Open Collaborative Network: a technological view}

In the last two decades the design of information systems for distributed organizations has undergone a paradigm shift, from data/message-orientation to process-orientation, giving to organizational context an important role. Modern Business Process Management Systems (BPMS) aim to support operational processes, referred to as workflows. BPMS can be efficiently realized using a Service-oriented Architecture (SOA), where the information system can be seen as a set of dynamically connectable services with the processes as the "glue" (Sun et al., 2016, Liu et al., 2009). The fit between BPMS and SOA has been formalized by the Business Process Model and Notation (BPMN) standard (OMG 2011, van der Aalst 2009).

In classical Business Process Management (BPM), processes are orchestrated centrally by the organization, and deployed for execution by predefined subjects internal to the organization. This closed-world approach is not suitable for OCN, where the open and collaborative nature of the global processes is essential. Other requirements may be incorporated, such as transparency control, easy participation, activity distribution, and decision distribution (Brambilla, 2011a). Thus, a certain level of control in knowledge flow is essential. Unfortunately, structural 

Furthermore, business requirements change frequently, not only for different enterprises but also for different period of time in the same enterprise, as markets and business practices change (Wang 2005, Sarnikar 2007). To add adaptation capabilities to the network-based social collaboration, some interesting works have been done on the formal modeling of collaboration processes as a negotiation, such as those based on Social BPM (Brambilla, 2011a), and Social Protocols (Picard, 2006). However, much work still has to be done before such approaches can be used on a regular basis.

BPMN is increasingly adopted in research projects as a language to specify guidelines for virtual organizations. For example in the ECOLEAD project (Romero and Molina, 2009; Peñaranda Verdeza et al. 2009) the BPM centric approach has been used to define a set of general and replicable business processes models for future instantiations into specific virtual organizations, providing rationale of activities that should be carried out by a set of actors in order to achieve the expected business process results. The ECOLEAD architecture presented in (Rabelo et al., 2006; Rabelo et al., 2008) is made of different services: (i) horizontal services, such as mailing, chat, task list, file storage, notification, calendar, wiki, forum, etc. (ii) basic services, such as security, billing, service composition, reporting, discovery; (iii) platform-specific services; (iv) legacy systems. The design approach is bottom-up, and it has been based on the web-services technology. From the technological point of view, such architecture is important as it contains elements that are incorporated into the current generation of $\mathrm{CN}$, which can be implemented in a diversity of platforms, equipment and devices.

In this paper we adopt a top-down design approach, focused on technological enablers of business logic. An enabler is a factor addressing a critical aspect, which is not already incorporated in existing approaches. More precisely, we propose a comprehensive approach for creating business logic integration solutions in OCN. A system architecture has been also implemented and demonstrated experimentally. The approach is based on three core technological enablers, providing a conceptual structure to design an OCN.

The first technological enabler is the workflow design, which provides coordination and flexibility in process. The workflow represents the sequence of steps, decisions, and the flow of work between the process participants (Ray and Lewis, 2009). We assume that the process model is encoded in BPMN, an open and standard language which in turn can be deployed and executed by a BPMS to directly control the workflow engines (Sharp 2012, Fraternali, 2011, Picard 2010).

The second technological enabler is the business rule design, which regulates how knowledge or information in one form may be transformed into another form through derivation rules. A derivation can either be a computation rule (e.g. a formula for calculating a value) or an inference rule (e.g. if some fact is true, then another inference fact must also be true) (Erikson 2000). Business rules are designed in terms of modular tasks and encapsulated into BPMN business rules tasks. To represent inference business rules, we used the de-facto standard for semantic rules on the web, Semantic Web Rule Language (SWRL)(W3C 2004). SWRL rules can be connected to facts expressed in Resource Description Framework (RDF) (W3C 2014) and to classes expressed 
into flexible logical sets (Wang 2005, Meech 2010). Business rules modeling and execution is an important application of the Semantic Web in collaborative environments (Meech 2010).

401 The third technological enabler is the privacy-preserving collaborative analytics. With regards to it, a workflow model is also used to assemble data flow together while preserving each individual flow. To maximize the usability of data flow without violating its market value, a suitable data perturbation technique is proposed, enabling collaborative analytics. Indeed relevant marketing concerns largely prevent data flow in collaborative networks. More specifically, business data is perturbed via digital stigmergy, i.e., a processing schema based on the principle of self-aggregation of marks produced by data. Stigmergy allows protecting data privacy, because only marks are involved in aggregation, in place of actual data values. There are two basic features which allow stigmergy to protect data flows in OCN. The first is the decentralization of control in decision making: each member has a partial view of the process which is insufficient to make the decision. Second, members are not statically organized but can dynamically move between different virtual enterprises.

In terms of supporting information technology, the combination of the first two enablers can support life cycle maintenance when managing process improvement and dynamic process 2009), context-aware BPs and self-adaptation of BPs (Cimino and Marcelloni, 2011). More specifically: (ii) the BPMN 2 specification includes a number of constructs and design patterns to model decentralized business-collaborations (Bechini et al., 2008); (i) the service-oriented computing, which is at the core of the BPMN 2 conception, is purposely designed to provide flexible, dynamic, component-oriented interoperability, for the dynamic composition of business application functionality using the web as a medium (Cimino and Marcelloni, 2011). However, the web services framework offers a low level of semantics for the specification of rich business processes, which is important for interoperability (Grefen et al., 2009). In the literature, considerable work employs Semantic Web as a prominent technique for semantic annotation of Web Services (Zeshan and Mohamad, 2011). With the help of well-defined semantics, machines can understand the information and process it on behalf of humans, as software agents (Cimino and Marcelloni, 2011). Furthermore, Semantic Web is at the core of context-awareness based modeling, where two levels can be distinguished to improve reusability ad adaptability: the service level and the external environment or context level (Furno and Zimeo 2014).

Given the above enablers, both the proposed approach and the prototype are referred to as DLIWORP: Distributed Business Logic Integration via Workflow, Rules and Privacy-preserving analytics. To better characterize the DLIWORP approach from a functional standpoint, the next section illustrates a pilot scenario, which will be employed to explain all the functional modules of the system. 


\section{Enacting Open Collaborative Network: a functional view through a pilot scenario}

\subsection{A pilot scenario of business collaboration}

As an example of business collaboration, let us consider the pilot scenario of Figure 2, concerning the design and the implementation of machinery. The scenario comes from a realworld case that has been established in a project named "PMI 3.0".

Here, the participants involved in the business are represented on the left: the client, the mechanical and the electrical firms. Both design and development activities, represented in the middle, are made of two main tasks: a mechanical task and an electrical task, carried out by the two respective firms. Finally, the management activity, which is represented on the right, consists in the coordination of the participants and in the orders planning tasks. With regard to the orders planning, each company schedules tasks on the basis of its own private business rules.

PARTICIPANTS

CLIENT

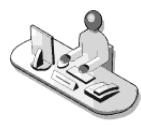

MECHANICAL FIRM

ELECTRICAL FIRM

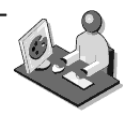

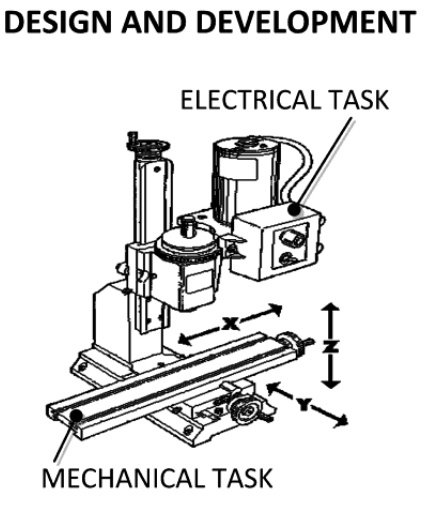

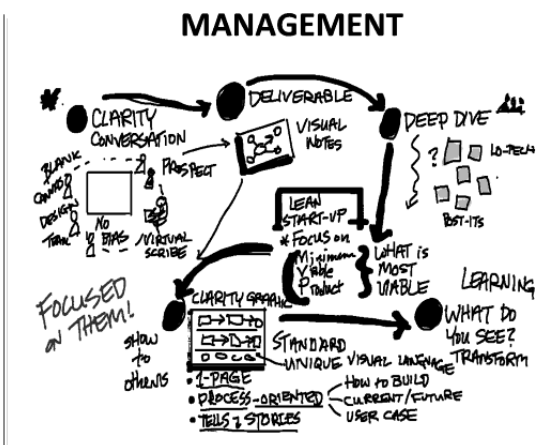

COORDINATION + ORDERS PLANNING TASK

Figure 2. Business collaboration: representation of a pilot scenario related to making machinery.

An order type can be either standard or innovative, i.e., an order very similar or completely different with respect to the past orders, respectively. An order can be performed either in the short or in the long period, depending on the following of factors: the order type, the number of "in progress" orders, the payment time, and the residual production capacity. The coordination task consists in conducting an iterative communication between the client and the firms, whose result is the order's planning or its rejection.

\subsection{BPMN and workflow design}

In order to describe the workflow design phase of the DLIWORP approach, let us first introduce some basic BPMN elements. To describe business processes, BPMN offers the Business Process Diagram (BPD). A BPD consists of basic elements categories, shown in Figure 3 and hereunder described from left to right. Events are representations of something that can happen during the business process; business flow is activated by a start event and terminated by an end event, while intermediate events can occur anywhere within the flow. BPMN offers a set of 
specialized events, such as the send/receive message events. Gateways represent decision points to control the business flow. The exclusive and the parallel gateway create alternative and concurrent flows, respectively. A pool is a participant in a business process, enclosing his workflow. An atomic business activity is a task. Different task types are allowed, and represented with different icons. The Control flow shows the order of execution of activities in the business process, whereas the message flow represents messages exchanged between business subjects.

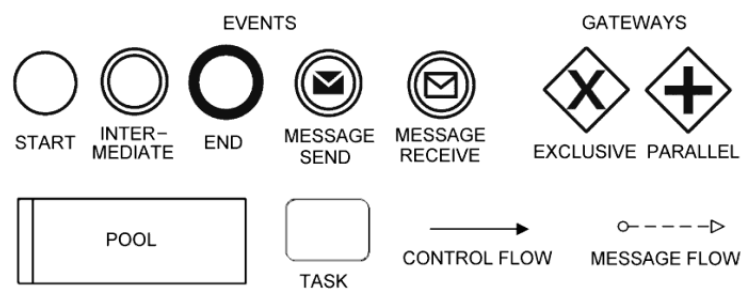

Figure 3. Basic BPMN elements: events, gateways, pool, task, flows.

Figure 4 shows a BPMN process diagram of the pilot scenario, consisting in the collaborative planning of an order. The start event in the Client pool indicates where the process starts, with a new order created in a user task, a task performed with the help of a person. A message with the order is sent from the client to the Shared Order Planning System, called hereafter "Planning System" for the sake of brevity. The Planning System splits the order into two parts, i.e. a mechanical and an electrical part, and sends them to the mechanical and electrical firms, respectively. Then, each firm performs its planning, represented as a business rule task, i.e., a specific BPMN task type. In a business rule task, one or more business rules are applied in order to produce a result or to make a decision, by means of a Business Rule Management System (BRMS) which is called by the process engine. The BRMS then evaluates the rules that apply to the current situation.
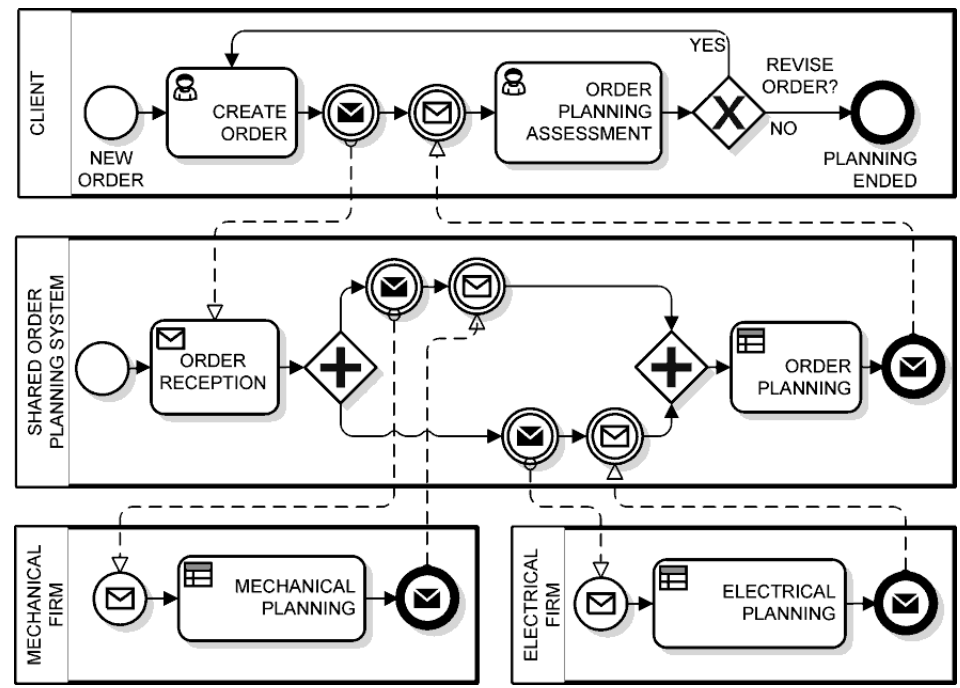

Figure 4. A simplified BPMN Process diagram of the collaborative planning of an order.

It is worth noting that each pool of a firm is supposed to be executed in a firm's private server, whereas the Planning System and the Client pools are supposed to be executed in a shared server. 
This way, the business rules of each firm are completely hidden to the Community. The decision of each firm is then sent to the Planning System, which carries out a logical combination via another business rule task, i.e., Order Planning, providing the Client with the overall planning of the order. Subsequently, the Client receives the planning and performs an assessment of it. The planning can either be revised, by creating a new order, or accepted, which causes the end of the workflow.

The next section covers the business rules design, i.e., how a business rule task is designed and implemented.

\subsection{Semantic Web and business rules design}

An ontological view of the collaborative planning of an order is represented in Figure 5, where base concepts, enclosed in gray ovals, are connected by properties, represented by black directed edges. More formally, a Client creates a New Order, which is characterized by a type (which can assume the value "standard" or "innovative"), a term (which can assume the value "short" or "long") and a payment (which can assume the value "fast" or "slow"). The new order is made of Work Modules. Work module is a generalized and abstract concept, i.e., it cannot be instantiated. In figure, the name of abstract concepts is represented with italic style. Mechanical Module and Electrical Module are work modules specialized from Work Module. In figure, specialized concepts are shown with white ovals and are connected by white directed edges to the generalized concept. Each module is characterized by a term (which can assume the value "short" or "long"), and is implemented by a Mechanical or Electrical Firm, respectively. Each firm inherits two properties from the generalized concept Firm. A firm has an in progress orders and retains a Residual Production Capacity. Both properties can assume the value "true" or "false".

The Ontology represented in Figure 5 can be entirely defined by using OWL, which is characterized by formal semantics and RDF/XML-based serializations for the Semantic Web. More specifically, the RDF specification defines the data model. It is based on XML data types and URL identification standards covering a comprehensive set of data types and data type extensions. The OWL specification is based on an RDF Schema extension, with more functional definitions.

The business rules of each participant can then be defined by using concepts of the Ontology and the structure of the SWRL is in the form of "horn clauses", following the familiar condition/result rule form. For the sake of brevity, in the scenario the ontology is globally shared between participants and the business rules are different for each participant. However, the ontology can be also modularized, to avoid sharing private concepts. 


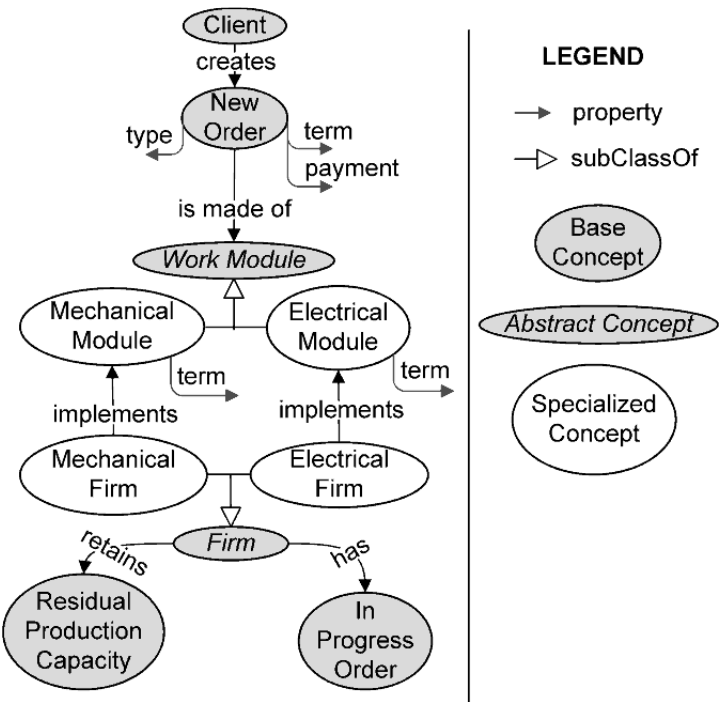

524 Figure 5. An ontological view of the collaborative planning of an order.

More specifically, the business rules can be informally expressed as follows:

(i) a mechanical firm places a new order in the short term if its type is standard and there are no in-progress orders; otherwise the order is placed in the long term;

(ii) an electrical firm places a new order in the short time if there is a residual production capacity and the payment is fast or if the payment is slow and its type is standard;

(iii) the planning system places a new order in the short term only if both modules have been placed in the short term.

Figure 6 shows the above knowledge in a natural language, via if-then rules.

An example of formal business rules expressed in SWRL is shown in Figure 7, in the human readable syntax, which is commonly used in the literature with SWRL rules and in rule editor

536 GUI. In this syntax: the arrow and the comma represent the then and the and constructs, 537 respectively; a variable is indicated prefixing a question mark; ontological properties are written in

538 functional notation. In the example of in Figure 7, each property can be found in the ontology of 539 Figure 5. 


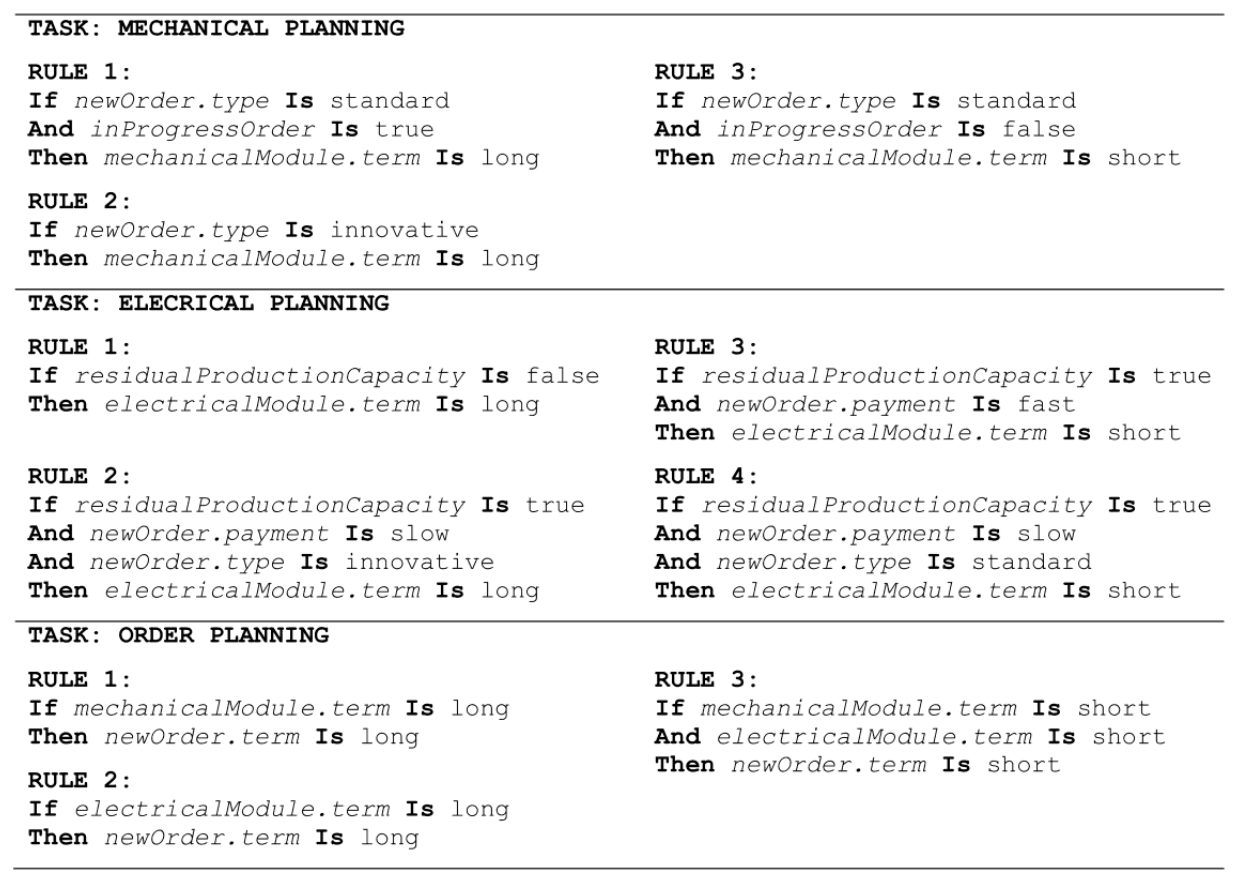

Figure 6. Business rules for each task of the collaborative planning of an order, expressed in natural language.

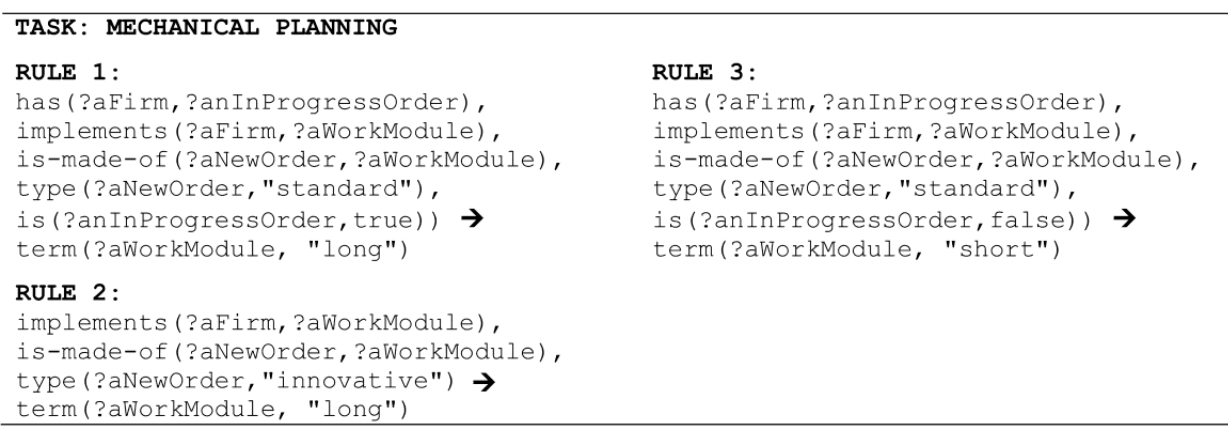

Figure 7. An example of formal business rules expressed in SWRL, using the human readable syntax.

The next section covers the business rules design, i.e., how a business rule task is designed and implemented.

\subsection{Stigmergy and privacy-preserving collaborative analytics}

Business rules are usually designed according to goals which are measurable via related Key Performance Indicators (KPIs), for each company and for the community itself. For this reason, the usability of the data flow connected to the workflow is a fundamental requirement. In a collaborative network the computation of KPIs must preserve the marketing value of data source to be aggregated, avoiding industrial espionage between competitors. In this section, we show the collaborative analytics technique designed for the DLIWORP approach.

Well (2009) defined formally the term collaborative analytics, as "a set of analytic processes where the agents work jointly and cooperatively to achieve shared or intersecting goals". Such 
processes include data sharing, collective analysis and coordinated decisions and actions. Collaborative analytics, while encompass the goals of their conventional counterparts, seek also to increase visibility of important business facts and to improve alignment of decisions and actions across the entire business (Well, 2009; Chen et al., 2012).

The focus here is not on specific KPIs: the technique is suitable for any business measurements that need to be aggregated handling company's data.

The problem in general can be brought back to comparing providers' performance. In practice, a collective comparison is related to the "to share or not to share" dilemma (Figure 8), an important reason for the failure of data sharing in collaborative networks.

\section{The "to share or not to share" dilemma}

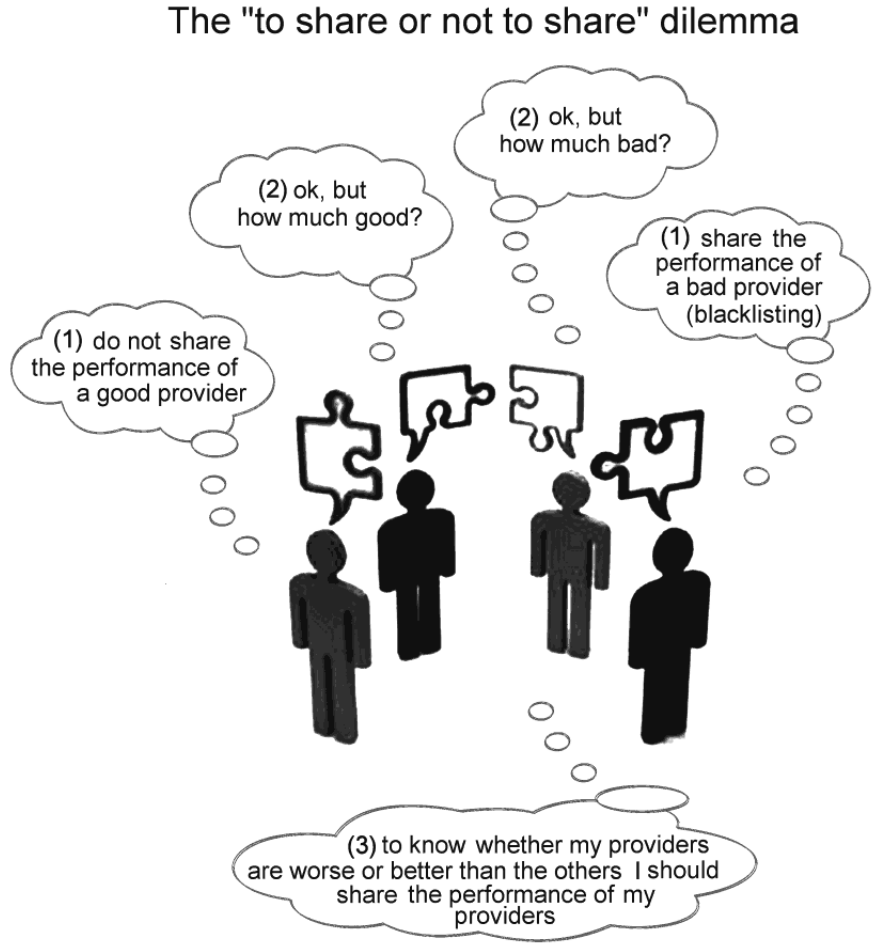

Figure 8. A representation of the "to share or not to share" dilemma between a group of buyers.

In the dilemma, a typical buyer does not like to share the performance of his good providers (keeping a competitive advantage over its rivals) and likes to share the performance of a bad provider (showing his collaborative spirit). However, each buyer knows a subset of the providers available on the market. The fundamental question of a buyer is: how much are my providers good/bad? To solve this question, providers' performance should be shared. This way, buyers with good providers would lose the competitive advantage. Given that nobody knows the absolute ranking of his providers, to share this knowledge is risky and then usually it does not happen.

In the literature, this problem is often characterized as "Value System Alignment" (Macedo et al., 2013). Values are shared beliefs concerning the process of goal pursuit and outcomes, and depend on the standard used in the evaluation. An example of value model is the economic value of objects, activities and actors in an e-commerce business. There are a number of methodologies and ontologies to define value models supporting BPs (Macedo et al. 2013). CN are typically formed by heterogeneous and autonomous entities, with different set of values. As a result, to 
identify partners with compatible or common values represents an important success element. However, tools to measure the level of alignment are lacking, for the following reasons: (i) the collection of information to build a model can be very difficult; (ii) the models are not easy to maintain and modify; (iii) if there are many interdependencies between values, the calculation becomes very time consuming because often it demands a record of past behavior that might not be available. Generally speaking, the approaches proposed for value system alignment are knowledge-based and belong to the cognitivist paradigm (Avvenuti et al. 2013). In this paradigm, the model is a descriptive product of a human designer, whose knowledge has to be explicitly formulated for a representational system of symbolic information processing. It is well known that knowledge-based systems are highly context-dependent, neither scalable nor manageable. In contrast to knowledge-based models, data-driven models are more robust in the face of noisy and unexpected inputs, allowing broader coverage and being more adaptive. The collaborative analytics technique based on stigmergy proposed in this paper is data-driven, and takes inspiration from the emergent paradigm. In this paradigm, context information is augmented with locally encapsulated structure and behavior. Emergent paradigms are based on the principle of self-organization of data, which means that a functional structure appears and stays spontaneous at runtime when local dynamism in data occurs (Avvenuti et al. 2013).

More specifically, our solution comes from perturbing business data via digital stigmergy. Stigmergy allows masking plain data by replacing it with a mark, a data surrogate keeping some original information. Marks enable a processing schema based on the principle of self-aggregation of marks produced by data, creating a collective mark. Stigmergy allows protecting data privacy, because only marks are involved in aggregation, in place of original data values. Moreover, the masking level provided by stigmergy can be controlled so as to maximize the usability of the data itself.

Let us consider an extension of the pilot scenario, with a new behavior in the workflow of 610 Figure 4: when the mechanical or the electrical planning does not satisfy the client requirements, 611 the Planning System must be able to select an alternative partner. To achieve this extension, an 612 Order Planning Assessment activity should be carried out by the Planning System too. Then, 613 another activity, called Select Alternative Partner, should compare partners' performance to carry 614 out a selection. Such performance must be made available by a collaborative analytics process.

615 Figure 9 shows an example of data flow designed to implement a privacy-preserving 616 collaborative analytics process in the DLIWORP approach. The Collaborative Analytics System

617 (called hereafter "System" for the sake of brevity) is the main pool located on a shared server and 618 coordinating pools of registered buyers. Each buyer's pool is located on a private server. 


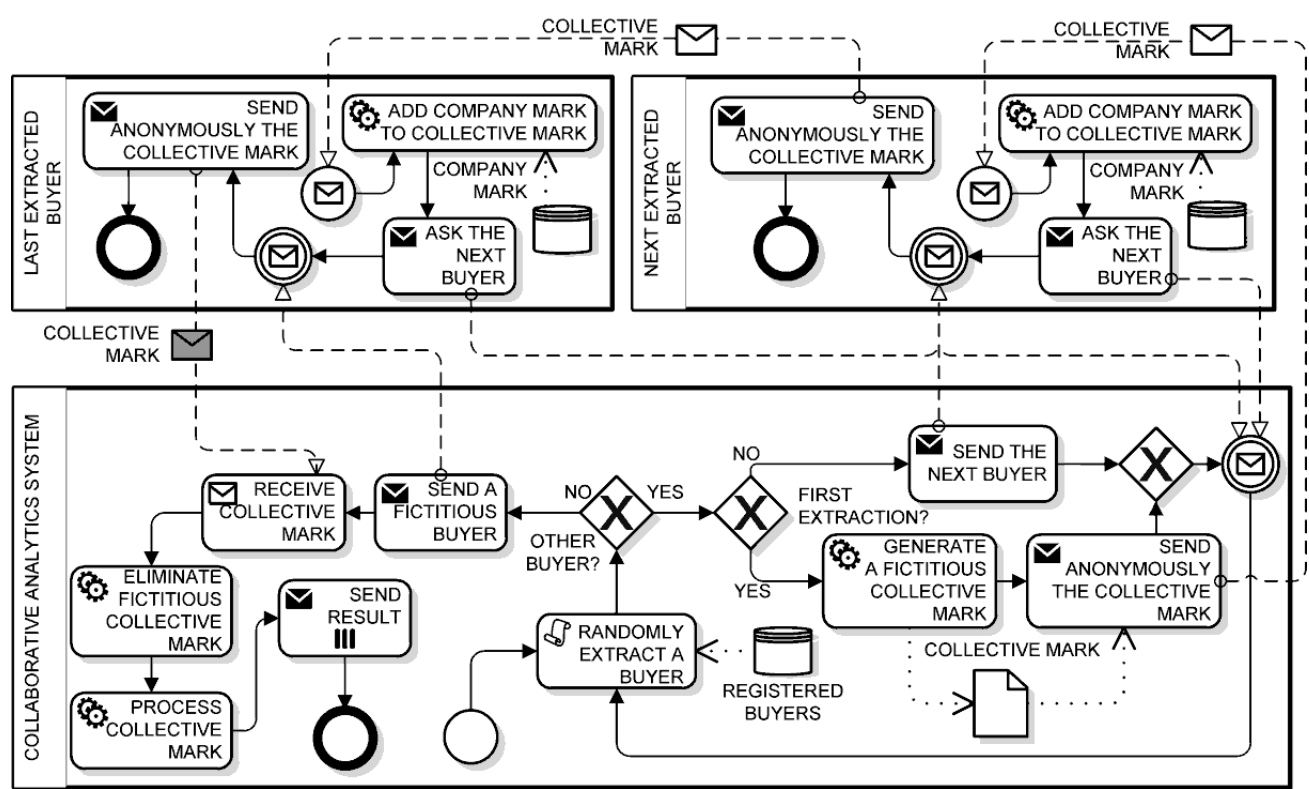

Figure 9. DLIWORP approach: an example of collaborative analytics using marker-based stigmergy to preserve individual data source.

The main goal of the data flow is to create a public collective mark by aggregating buyers' private marks. This aggregation process protects buyers' mark from being publicized. More specifically, at the beginning the System randomly extracts a buyer and generates a fictitious collective mark. A fictitious mark is a mark created from artificial data that mimics real-world data, and then cannot be distinguished from an actual mark in terms of features. The collective mark is then anonymously sent to the extracted buyer, who adds his private mark to it and ask the System for the next buyer. The system will answer with a randomly extract next buyer. Then, the buyer sends anonymously the collective mark. This way, the collective mark is incrementally built and transferred from a buyer to another one, under orchestration of the System. Each buyer is not aware of his position in the sequence. This is because the first extracted buyer receives a fictitious collective mark, and because the sender is always anonymous. The last extracted buyer will be provided with a fictitious buyer by the system. Such fictitious buyer actually corresponds to the System itself. After receiving the collective mark, the System subtracts the initial fictitious mark, thus obtaining the actual collective mark, which is then processed (so as to extract some common features) and sent to all buyers. By comparing the collective mark with his private mark, each buyer will be able to assess his position with respect to the collective performance. The results of this process can be used by to select a partner whose performance is higher than the collective performance.

In the next section let us consider the marker-based stigmergy, which is the basis for the data perturbation and integration used in the DLIWORP approach.

\section{Using stigmergy as collaborative analytics technique}


literature, the mechanisms used to organize these types of systems and the collective behavior that emerges from them are known as swarm intelligence, i.e., a loosely structured collection of interacting entities (Avvenuti et al. 2013; Gloor, 2006; Bonabeau et al., 1999). In our approach, the stigmergic mechanism has been designed as a multi-agent system. Software agents are a natural metaphor where environments can be modeled as societies of autonomous subjects cooperating with each other to solve composite problems (Cimino et al. 2011). In a multi-agent system, each agent is a software module specialized in solving a constituent sub-problem.

The proposed a collaborative analytics mechanism is based on two types of agents: the marking agent and the analytics agent, discussed in the next section.

\subsection{The Marker-based Stigmergy}

Let us consider a real value - such as a price, a response time, etc. - recorded by a firm as a consequence of a business transaction. As discussed in Section 3, to publicize the plain value with the associated context may provide advantages to other firms over the business competition. In this context, data perturbation techniques can be efficiently used for privacy preserving. In our approach a real value is represented and processed in an information space as a mark. Thus, marking is the fundamental means of data representation and aggregation. In Figure 10 the structure of a single triangular mark is represented. Here, a real value $x_{j}$, recorded at the time $t$ by the $j$-th firm, is represented with dotted line as a mark of intensity $I(t)(x)$ in the firm's private space. A triangular mark is characterized by a central (maximum) intensity $I_{M A X}$, an extension $\varepsilon$, and a durability rate $\theta$, with $\varepsilon>0$ and $0<\theta<1$, where $\varepsilon$ and $I_{M A X}$ are the half base and the height of the triangular mark, respectively.

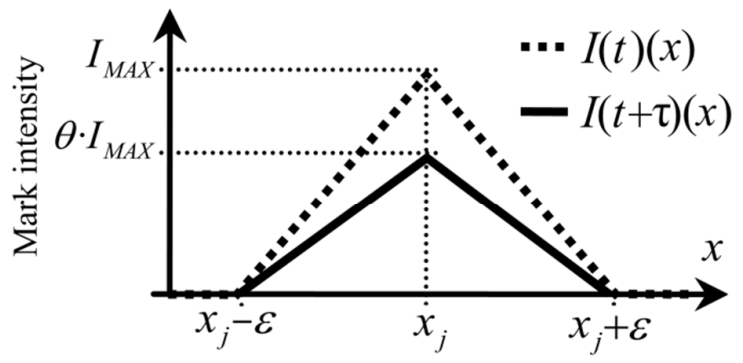

Mark position and extension

Figure 10. A single triangular mark released in the marking space by a marking agent (dotted line), together with the same mark after a temporal step (solid line).

Figure 10 shows, with a solid line, the same mark after a period $\tau$. In particular, the mark intensity spatially decreases from the maximum, corresponding with the recorded value $x_{j}$, up to zero, corresponding with the value of $x_{j} \pm \varepsilon$. In addition, the intensity released has a durability rate, $\theta$, per step, as represented with the solid line. More precisely $\theta$ corresponds to a proportion of the intensity of the previous step. Hence, after a certain decay time, the single mark in practice disappears.

Let us consider now a series of values, $x_{j}^{(t)}, x_{j}^{(t+\tau)}, x_{j}^{(t+2 \tau)}, \ldots$, recorded by a firm as a consequence of a series of business transactions. Marks are then periodically released by marking agents. Let us suppose that each firm has a private marking space and a private marking agent. The 
decay time is longer than the period, $\tau$, by which the marking agent leaves marks. Thus, if the company holds very different values in the series, the marking agent releases marks on different positions, and then the mark intensities will decrease with time without being reinforced. If the company holds an approximately constant value, at the end of each period a new mark will superimpose on the old marks, creating a lasting mark. More formally it can be demonstrated that the exact superimposition of a sequence of marks yields the maximum intensity level to converge to the stationary level $I_{M A X} /(1-\theta)$ (Avvenuti et al. 2013). For instance, with $\theta=0.75$ the stationary level of the maximum is equal to $4 \cdot I_{M A X}$. Analogously, when superimposing $N$ identical marks of different companies, we can easily deduce that the intensity of the collective mark grows with the passage of time, achieving a collective stationary level equal to $N$ times the above stationary level.

Figure 11 shows four private marks (thin solid lines) with their collective mark (thick solid line) in three different contexts, created with $I_{M A X}=10, \varepsilon=0.3, \theta=0.75$. In Fig (a) the private marks have a close-to-triangular shape, with their maximum value close to $I_{M A X} /(1-\theta)=4 \cdot I_{M A X}=$ 40. It can be deduced that, in the recent past, record values were very close and almost static in the series. As a consequence, also the collective mark has a shape close to the triangular one, with a maximum value close to $N \cdot 40=160$. We say reference private marks and reference collective mark when marks are exactly triangular, because they produce the highest marks. Figure 11 (b) shows a sufficiently static context, where record values in the recent past were not very close and not very static. For this reason, private marks have a rounded-triangular shape and the collective mark has a Gaussian-like shape. Finally, Figure 11 (b) shows an actual market context, where private and collective marks are very dynamic.

The first important observation is that Figure 11 (a) and Fig (b) do not present privacy problems, because all companies have similar performance. i.e., their providers are equivalent. In Figure 11 (c) there is dynamism but also a structural difference between companies: two of them have better performance. Here, the reference private marks and the reference collective mark are also shown, with dashed lines and located at the barycenter of the collective mark. It is worth noting that the contrast between marks and reference marks is a quite good indicator of the position and the dynamism of each company in the market. The two best companies are at the right of the reference private mark. Furthermore, all companies are in a dynamic context, because the shape of their marks is far from the triangular one. Finally, comparing the shapes of the reference collective mark and the collective mark, it can be also deduced the amount of overall dynamism.

We can associate some semantics to the parameters of a mark. A very small extension $(\varepsilon \rightarrow 0)$ and a very small durability rate $(\theta \rightarrow 0)$ may generate a Boolean processing: only almost identical and recent records can produce collective marking. More specifically to increase the extension value implies a higher uncertainty, whereas to increase the durability value implies a higher merging of past and new marks. A very large extension $(\varepsilon \rightarrow \infty)$ and a very large durability rate $(\theta \rightarrow 1)$ may cause growing collective marks with no stationary level, because of a too expansive and long-term memory effect. Hence, the perturbation carried out by stigmergy can be controlled so as to maximize the usability of the data itself while protecting the economic value of information. 


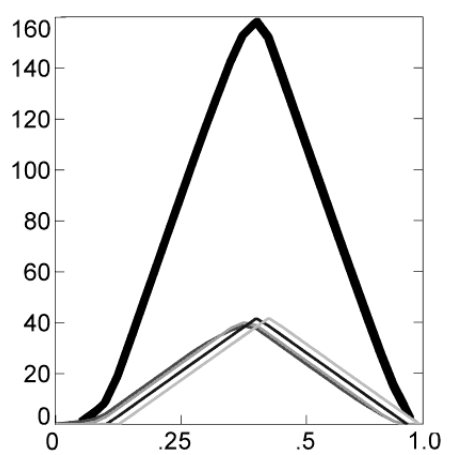

(a)

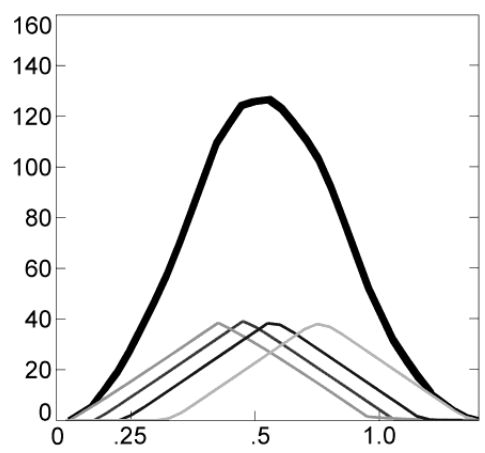

(b)

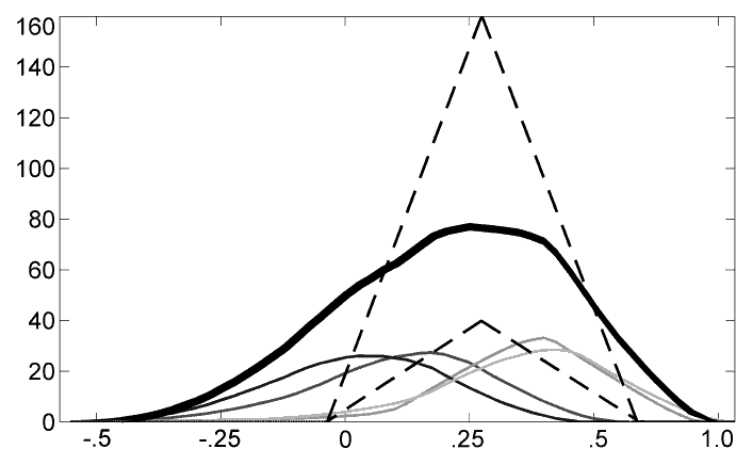

(c)

Figure 11. Four private marks (thin solid lines) with their collective mark (thick solid line) in different contexts: (a) very static; (b) sufficiently static; (c) dynamic with reference marks (dashed line). $I_{M A X}=10, \varepsilon=0.3, \theta=0.75$.

To summarize the approach, Figure 12 shows the classification of four recurrent patterns in marking, based on the proximity to a triangular shape and to a barycentric position of the mark (solid line) with respect to the reference mark (dashed line).

Exploiting the above observations, in the following, we discuss how a different type of agent can recognize the patterns of Figure 12: the analytics agent. Basically, the analytics agent is responsible for assessing the similarity and the integral difference of a mark with respect to the corresponding reference mark, as represented in Figure 13. More formally, given a reference mark, $A$, and a mark, $B$, their similarity is a real value calculated as the area covered by their intersection (colored dark gray in the figure) divided by the area covered by the union of them (colored light and dark gray). The lowest similarity is zero, i.e., for marks with no intersection, whereas the highest is one, i.e., for identical marks. The barycentric difference is the normalized difference between the right and the left areas of the mark with respect to the barycenter of the reference mark.

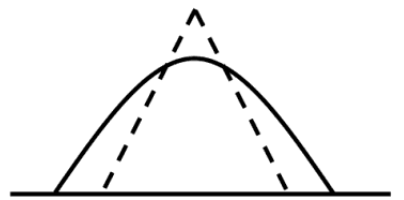

(a) stable and average performance

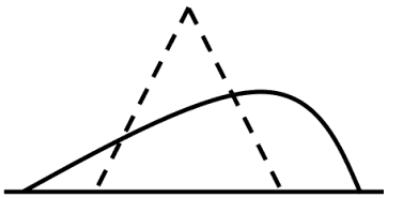

(b) variable and positive performance 


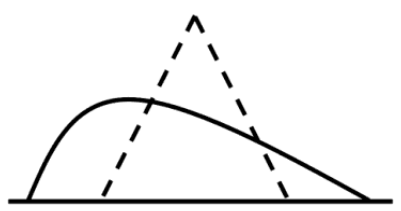

(c) variable and negative performance

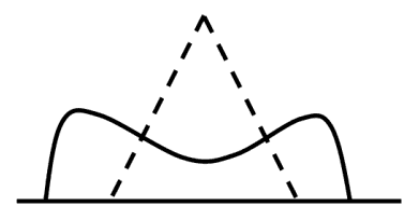

(d) very dynamic and balanced performance
739

740

741

742

Figure 12. Classification of four recurrent patterns in marking, based on the proximity to a triangular shape and to a barycentric position of the mark (solid line) with respect to the reference mark (dashed line).

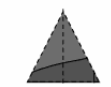

$|A|$

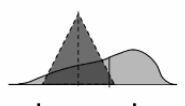

$|A \cup B|$

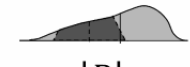

$|B|$

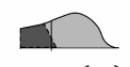

$\boldsymbol{S}=\frac{|A \cap B|}{|A \cup B|}$
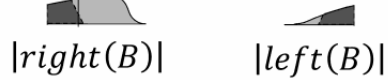

$\boldsymbol{D}=\frac{|\operatorname{right}(B)|-|\operatorname{left}(B)|}{|B|}$

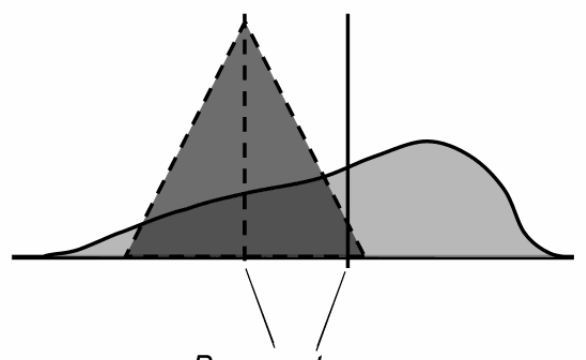

Barycenter

Figure 13. Representation of Similarity $(S \in[0,1])$ and barycentric Difference $(D \in[-1,1])$ of a mark (B) with respect to the corresponding reference mark (A).

Thus, the proximity to a triangular shape can be then measured by the similarity, whereas the barycentric position of the mark with respect to the reference mark can be assessed by means of the barycentric difference, as represented in Figure 14.

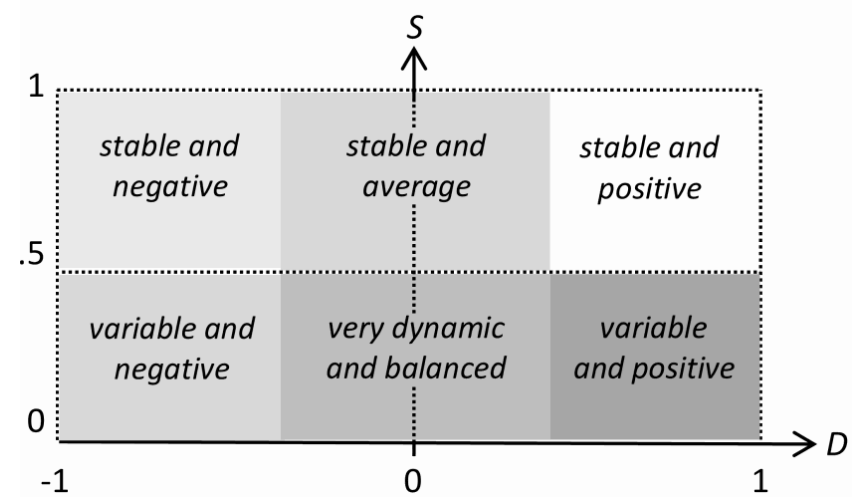

Figure 14. Analytics agent: classification of patterns on the basis of Similarity (S) and barycentric Difference (D).

\subsection{A numerical example of collaborative analytics based on stigmergy}

In section 4.4, we considered, in an extension of the pilot scenario, an activity called Select Alternative Partner, which compares partners' performance to carry out a selection. Such performance can be made available by a collaborative analytics problem. In this section we adopt e the KPI productivity as an example of partners' performance, and we show a numerical example of processing of such KPI, performed by the marking agent and the analytics agent. The numerical 
example is based on the publicly available dataset Belgian Firms ${ }^{1}$, containing 569 records each characterized by four attributes: capital (total fixed assets), labour (number of workers), output (value added) and wage (wage cost per worker) (Verbeek, 2004). Starting from raw data, the KPI productivity has been first calculated as output divided by labour. Then, 7 clusters representing provider companies have been derived by using the Fuzzy C-Means algorithm. Subsequently, 4 buyers have been supposed, and each buyer has been connected to three providers.

Figure 15 shows the output of the marking agent in terms of private marks (solid gray lines), collective mark (solid black line), and reference marks (dotted lines), with different extension values: (a) $\varepsilon=30$ for all buyers; (b) $\varepsilon=60$ for B1 and $\varepsilon=30$ for the others. In the figure, the buyer B1 has been highlighted with a larger thickness. It can be noticed that the different extension values sensibly modifies the shape, and then the perturbation, of the buyer's private mark.

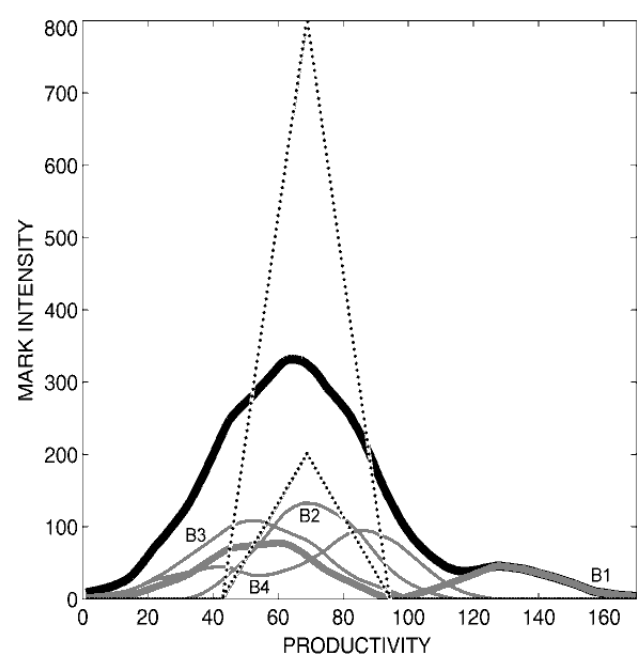

(a)

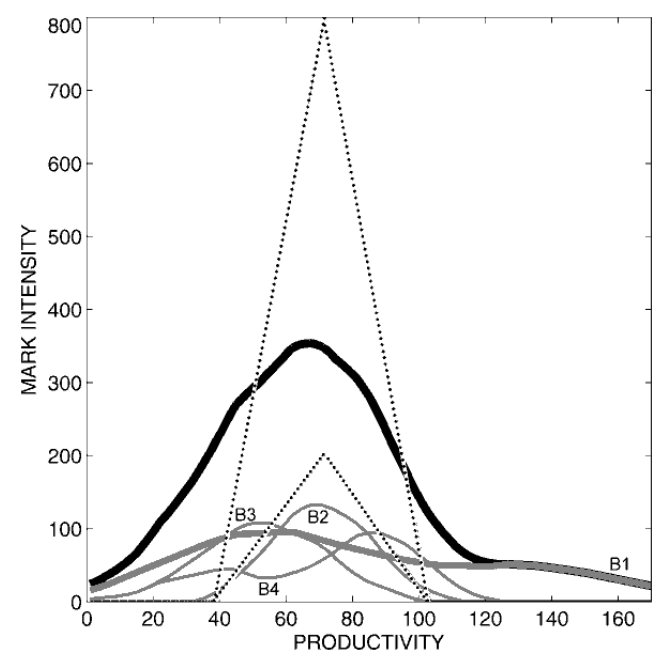

(b)

Figure 15. Belgian firms scenario: four buyers' private marks (solid gray lines), collective mark (solid black line), and reference marks (dotted lines), with different extension values: (a) $\varepsilon=30$ for all buyers; (b) $\varepsilon=60$ for the buyer B1 (with larger thickness) and $\varepsilon=30$ for the others.

Table 2 shows the patterns recognized by the analytics agent. It is worth noting that, despite the different level of perturbation that affected the buyer B1, there are no differences in the Performance patterns detected.

Table 2 Performance patterns of each buyer, with respect to Similarity (S) and barycentric Difference (D) for the Belgian Firms scenario.

\begin{tabular}{cccl}
\hline & S & D & Performance pattern \\
\hline B1 & 0.26 & -0.07 & dynamic and balanced \\
B2 & 0.73 & -0.08 & stable and average \\
B3 & 0.37 & -0.58 & variable and negative \\
B4 & 0.31 & -0.20 & dynamic and balanced \\
\hline
\end{tabular}

(a)

\begin{tabular}{cccl}
\hline & S & D & Performance pattern \\
\hline B1 & 0.32 & -0.03 & dynamic and balanced \\
B2 & 0.77 & -0.01 & stable and average \\
B3 & 0.36 & -0.64 & variable and negative \\
B4 & 0.39 & 0.15 & dynamic and balanced \\
\hline
\end{tabular}

(b)

\footnotetext{
${ }^{1}$ http://vincentarelbundock.github.io/Rdatasets/doc/Ecdat/Labour.html
} 


\section{Architecture, administration and experimentation of the supporting system}

This section focuses on the $\mathrm{OCN}$ as a system in its life-cycle. A prototypical system architecture for the DLIWORP approach has been developed and experimented under a research and innovation program supporting the growth of small-medium enterprises.

So far we have identified three technological enablers on the basis of initial requirements, and then we have defined standard specifications and technological solutions, addressing each of the factors. As a foundation of our approach, we require decomposition of modeling into workflow, business rules, and privacy-preserving collaborative analytics. An especially important point is that, if just one factor is not supported, then the other two factors cannot adequately foster the distributed business logic inherent in the OCN.

We have described our approach through a demonstrative scenario, to shows how information technology oriented solutions can be integrated towards the business perspective. The pilot scenario is representative of some other scenarios which have been developed and tested in the context of the regional research and innovation project. However, the scenario cannot be considered a reference case. Our main purpose is to show the ability of the approach to express aspects of interest that have been encountered in a real-world OCN. In the literature, the benefits of collaboration are clear, but it is also apparent that different paths to a successful collaboration can be envisaged, since many drivers exist and new ones tend to appear (Camarinha-Matos, 2014). Indeed, emergent behavior resides in keeping enterprises prepared to manage different kinds of business processes. This entails support for abstraction and modeling techniques in combination. Here, the notion of business process model provides a number of advantages to capture the different ways in which each case (i.e., process instance) in an OCN can be handled: (i) the use of explicit process models provides a means for knowledge sharing between community members; (ii) systems driven by models rather than code have less problems when dealing with change; (iii) it better allows an automated enactment; (iv) it better support redesign; (v) it enables management at the control level.

The remainder of this section is organized into three subsections, covering the system architecture, the system administration, and its experimentation, respectively.

\subsection{System architecture}

Figure 16 shows an UML (Unified Modeling Language) architectural view of an OCN supporting the DLIWORP approach. Here, device, execution environment and component are represented as dark gray cuboids, light gray cuboids, and white rectangles, respectively. Links between execution environments represent bidirectional communication channels, whereas usage relationships between components are specified by their provided and required interfaces, represented by the "lollypop" and "socket" icons, respectively. Finally, user roles are represented by the "stick man" icon. There are three device categories: Business Community Server, which is the computer(s) hosting data and services shared by the collaborative network; Company Server, which is a computer hosting data and services that must be kept private by each company; Client, 
821 which is a personal or office computer hosting client applications for users. There are two users

822 (roles): Business Worker, who is a participant to a workflow of the collaborative network; a

823 business worker uses the Web Browser as main execution environment; Business Logic Manager

824 is responsible for designing and deploying the business logic, via the DLIWORP approach; he

825 uses different client applications: a Stigmergic Modeler for designing data perturbation, a Semantic

826 Modeler for designing ontology and semantic rules, a Workflow Modeler for designing an

827 executable business collaboration, and a Business Analytics environment to access the

828 collaborative analytics. There can be many business workers and business logic managers for each

829 company. Both the Business Community Server and the Company Server have the following

830 execution environments: a Workflow Management System, where workflows are deployed (in the

831 Business Process Model knowledge base), executed (by the Workflow Engine), and recorded (by

832 the Event Repository database); a Semantic Web Service, hosting the Ontology and Rules

833 knowledge base and the Semantic Engine for executing business rule tasks; a Multiagent System

834 Manager, hosting the Marking Agent and the Analytics Agent, as well as their Marks Repository.

835 Specific point-to-point connections of the above execution environments in a network of

836 independent nodes should be avoided, because it hampers maintenance (Bechini et al. 2008). Thus,

837 the execution environments should be connected to an Enterprise Service Bus (ESB), a service-

838 oriented middleware for structural integration. For this purpose, the Content Based Routing

839 component provides a routing service that can intelligently consider the content of the information

840 being passed from one application to another, whereas the Transformation Services transform data

841 to and from any format across heterogeneous structure and data types. In addition, the latter

842 module can also enhance incomplete data, so as to allow execution environments of different

843 vendors to coexist. An ESB can also be connected to other ESBs, to allow an easy integration

844 between collaborative networks.

845 Moreover, the execution environment hosting the ESB hosts an Enterprise Service Portal 846 (ESP), a framework for integrating information, people and processes across organizational 847 boundaries. For this purpose, the Users Management, the Groups Management, and the Messages 848 Management components provide support for profiles, privileges, roles, workgroups, companies, 849 business messaging, etc. The Web Content Management component allows to create, deploy, 850 manage and store content on web pages, including formatted text documents, embedded graphics, 851 photos, video, audio, etc. The Records Management component allows managing what represents 852 proof of existence. Indeed, a record is either created or received by an organization in pursuance of 853 or compliance with legal obligations, in a business transaction. The Document Management 854 component is used to track and store documents, keeping track of the different versions modified 855 by different users (history tracking). Finally, the Content Repository component is the main store 856 of digital content shared by the above components. It allows managing, searching and accessing 857 sets of data associated with different services, thus allowing application-independent access to the 858 content. 


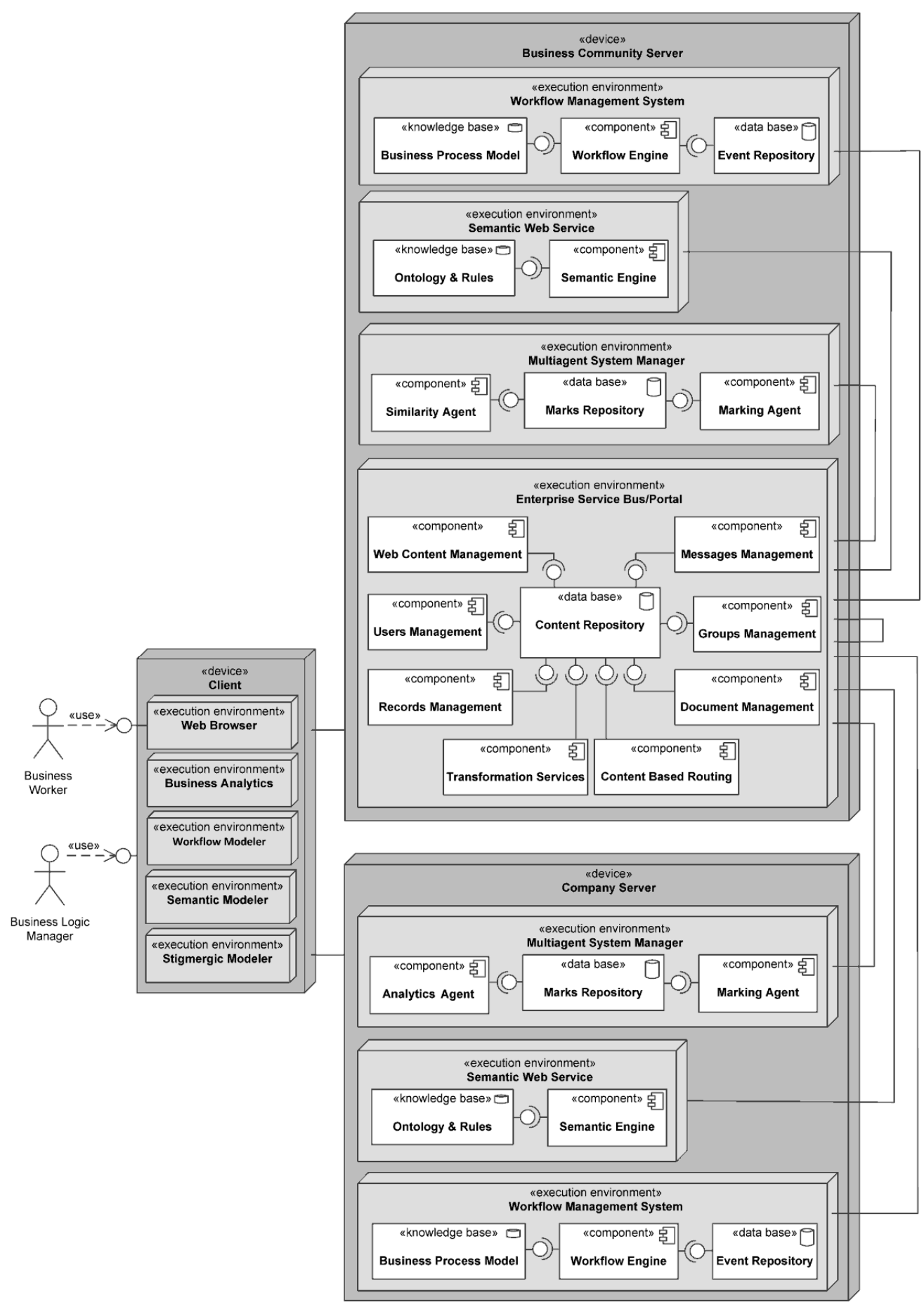

Figure 16. Overall architectural view of a OCN supporting the DLIWORP approach.

The System has been developed with public domain software, in order to be completely costless in terms of licenses for the firms joined to the research program. Table 3 lists the software products that have been considered. For each component, a comparative analysis has been carried out to choose the most fitting product, represented in boldface style in the table. The main features that have been taken into account in the comparative analysis are: full support with the standard languages (mostly BPMN 2.0 and SWRL); interoperability; free license and usability. 
Table 3 Software products compared for the DLIWORP system implementation. The product selected has been represented with boldface style.

\begin{tabular}{|c|c|c|}
\hline System component & Software product & Web Reference \\
\hline \multirow[t]{7}{*}{ Enterprise Service Portal } & Liferay & www.liferay.com \\
\hline & eXo platform & www.exoplatform.com \\
\hline & Alfresco & www.alfresco.com \\
\hline & Magnolia & www.magnolia-cms.com \\
\hline & Nuxeo & www.nuxeo.com \\
\hline & Jahia & www.jahia.com \\
\hline & Apache Lenya & lenya.apache.org \\
\hline \multirow[t]{5}{*}{ Workflow engine and modeler } & Kaleo & www.liferay.com \\
\hline & Activity & activiti.org \\
\hline & Aperte Workflow & www.aperteworkflow.org \\
\hline & BonitaBpm & www.bonitasoft.com \\
\hline & jBPM & www.jbpm.org \\
\hline \multirow[t]{4}{*}{ Semantic Engine and modeler } & Apache Stanbol & stanbol.apache.org \\
\hline & Apache Jena & jena.apache.org \\
\hline & Pellet & clarkparsia.com/pellet \\
\hline & Protegè & protege.stanford.edu \\
\hline \multirow[t]{2}{*}{ Multiagent System Manager } & Repast Symphony & repast.sourceforge.net \\
\hline & Jade & jade.tilab.com \\
\hline \multirow[t]{6}{*}{ Business Analytics } & Jaspersoft & community.jaspersoft.com \\
\hline & Alfresco Audit Analysis and Reporting & addons.alfresco.com \\
\hline & Alfresco Business Reporting & addons.alfresco.com \\
\hline & Pentaho & www.pentaho.com \\
\hline & QlikView & www.qlik.com \\
\hline & SpagoBI & www.spagobi.org \\
\hline
\end{tabular}

873

\subsection{System administration}

Each of the above system components has been configured or customized to support the major activities carried out by actors for achieving their expected business process results. This customization process mainly consists in (i) exposing functionalities essential for the user role and (ii) hiding functionalities that are not applicable. For this purpose, 71 overall use cases were determined in the analysis phase of the project. In what follows, the user-interface views of the key functions supported by the system are summarized, together with the most important use cases.

The Enterprise Service Portal shall support and facilitate 27 use cases, grouped into four categories: (i) actors management (including creation, modification, access and manipulation); (ii) membership and structure management; (iii) profiling and competency management (including collaborative rating); (iv) sharing and exchange of spaces, resources, messages, opinions for collaboration with actors, including following, searching, inviting actors, tagging. As an example, Figure 17 shows a web-based user interface of the Enterprise Service Portal, related to a technical document of a new order which was previously uploaded in an actor's library. The interface allows 

preferred.

\section{Dettagli documento}

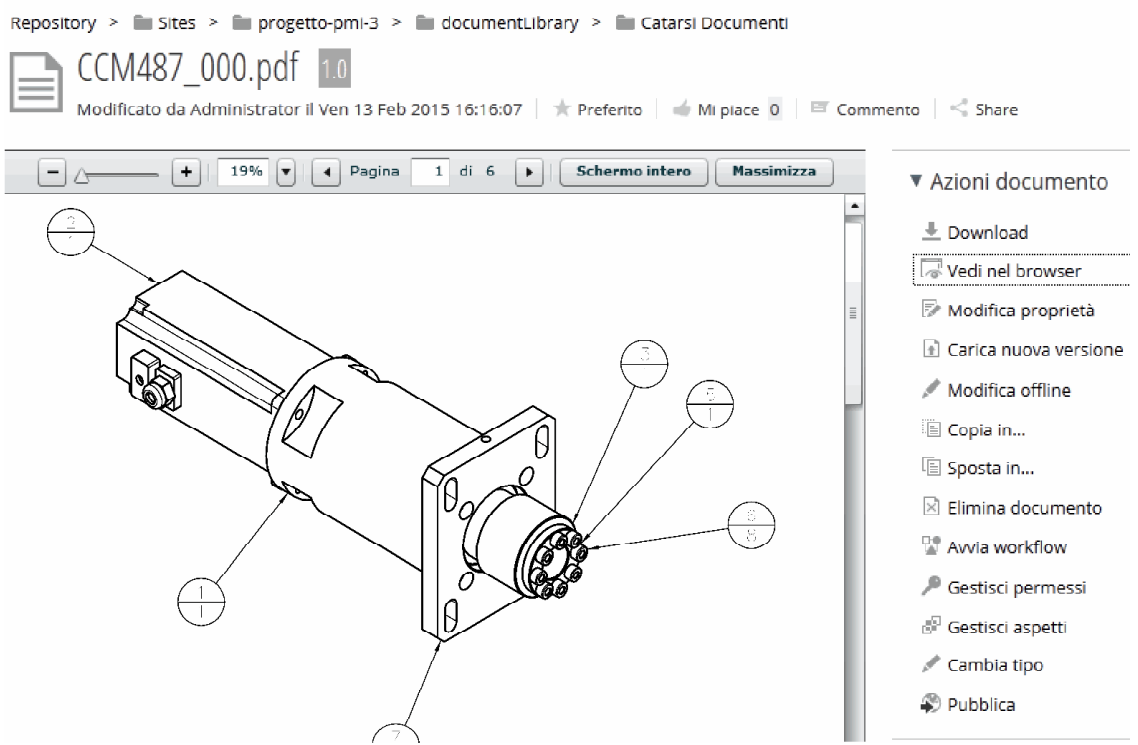

891

892

893

894

895

896

897

898

899

900

901

902

Figure 17. User interface view of the Enterprise Service Portal, created via Alfresco Community.

The Workflow engine and modeler supports and facilitates 11 use cases, belonging to four categories: (i) workflow management (including creation, selection, modification, access and manipulation); (ii) task management (select and carry out the next task, list the users who are eligible for performing a task, list the previous tasks); (iii) actors management (actor creation, assigning tasks to actors); (iv) data objects and storage management (data object creation, scope, flow). As an example, Figure 18 shows the user interface of the Workflow Modeler, with the editor providing a graphical modeling canvas and palette. A business process in BPMN 2.0 notation can be easily created, converted into XML, and deployed on the workflow engine. Deployment artifacts can be also imported into another Workflow Modeler.

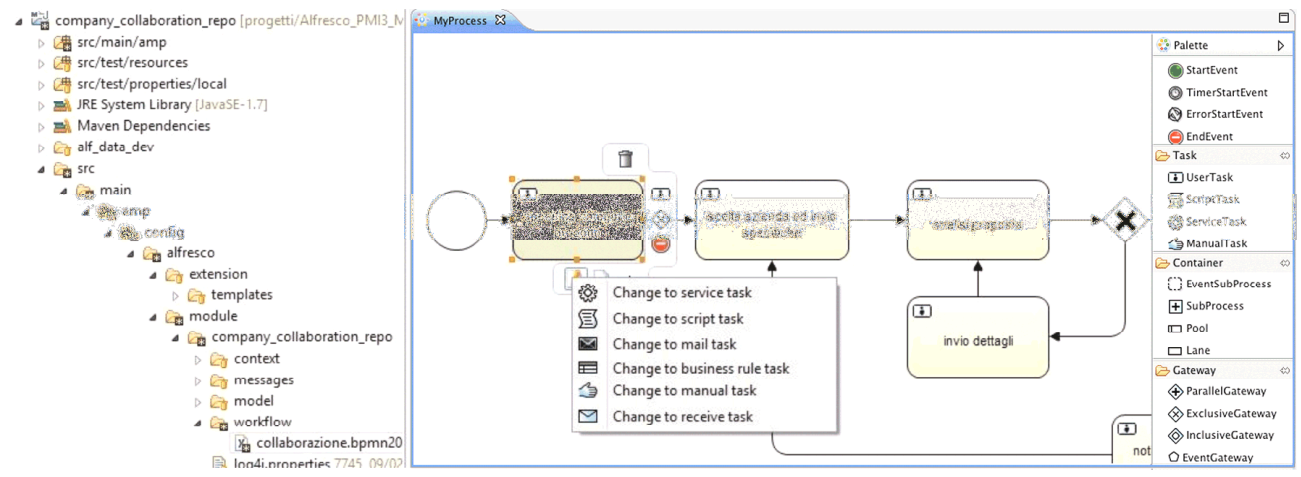

Figure 18. User interface view of the Workflow Modeler, created via Activity Designer.

The Semantic Engine and Modeler supports 9 use cases of three categories: (i) ontology 
selection, editing, deletion); (iii) engine management (apply ontology and rules). As an example, in Figure 19 the Semantic Modeler is shown. Here, the ontology of a collaborative planning of an order (modeled in Figure 5 and Figure 6) has been created. More specifically, the modeler allows (i) to organize concepts of the domain in classes and hierarchies among classes; (ii) to define the properties of the classes; (iii) to add constraints (allowed values) on the properties; (iv) to create instances; (v) to assign values to the properties for each instance.
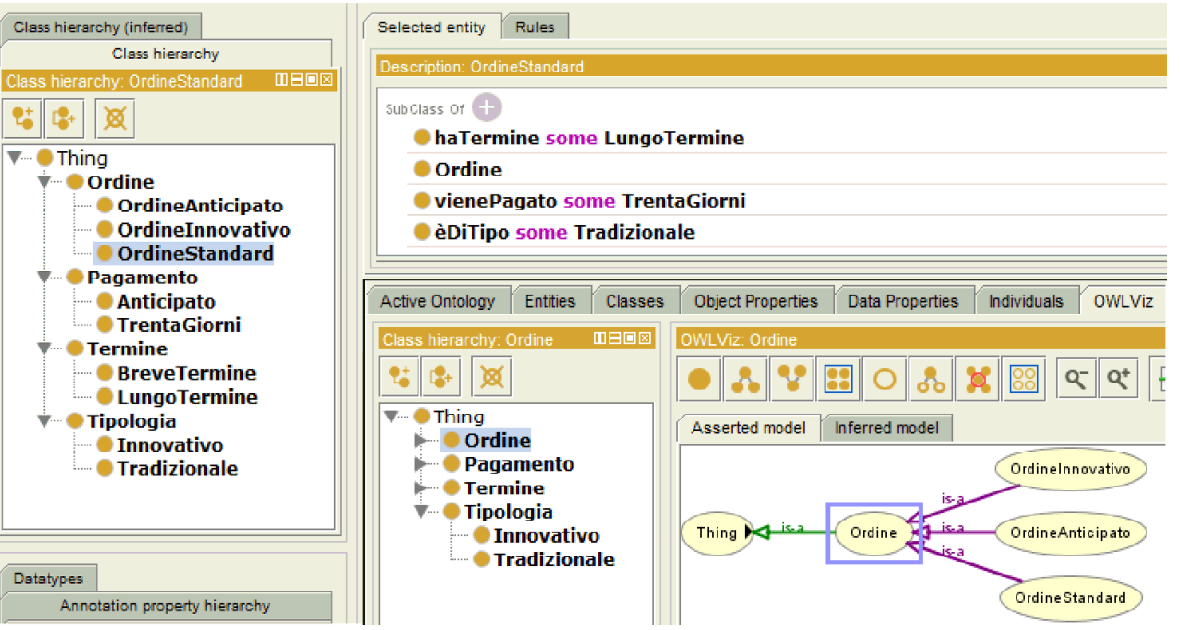

Figure 19. User interface view of the Semantic Modeler, created via Protégé.

The Multiagent System Manager supports 8 user cases, separated into the following categories: (i) marking agent management (agent creation, editing, deletion, execution, parameterization); (ii) analytics agent management (agent creation, editing, deletion, integration, execution, parameterization). Figure 20 shows the user interface view of the Multiagent System Manager, which allows starting, stopping and managing the stigmergic process carried out by the different agents. The panel provides also a configuration menu where to set the most important parameters, such as the durability (or evaporation) rate, mark extension, and mark maximum intensity.

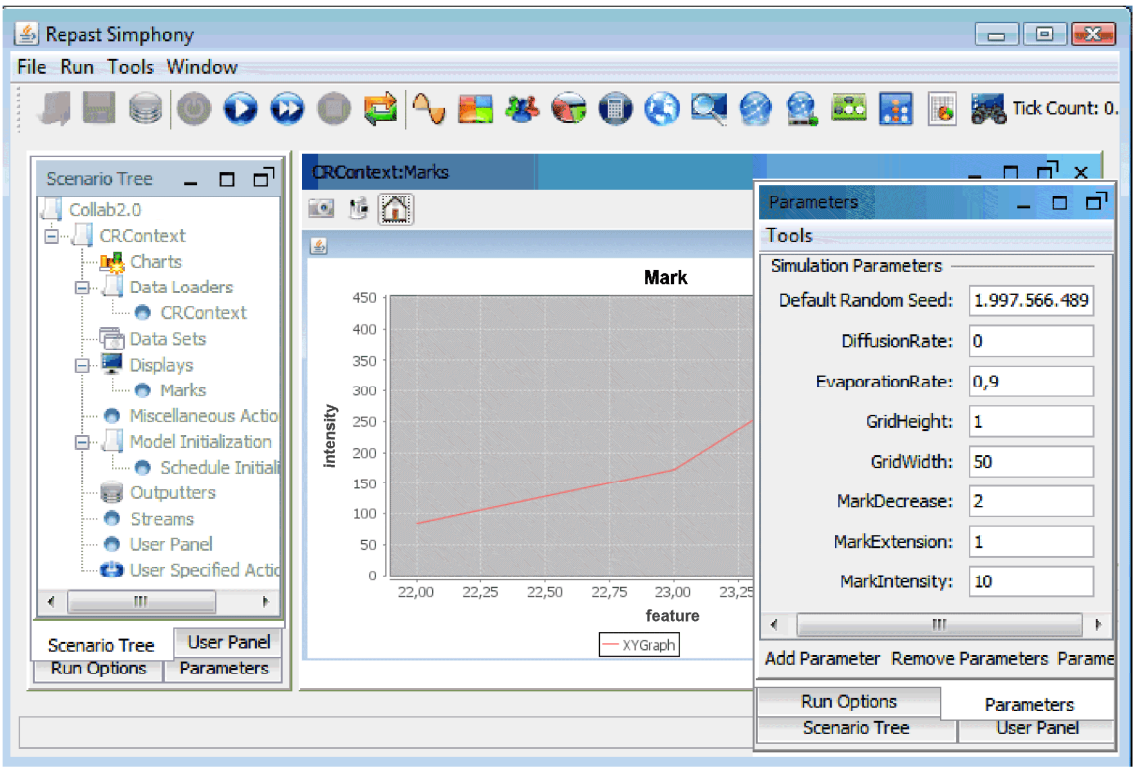

Figure 20. User interface view of the Multiagent System Manager, created via Repast Symphony. 
Finally, The Business Analytics component supports 16 use cases, organized into four categories: (i) report template management (template create, modify, remove, search); (ii) ETL (Extract, Transform and Load) procedure definition, modify, remove; (iii) report production schedule (definition, modify, remove); (iv) ad-hoc report management (create, show, export, search, remove); (v) dashboard management (create, edit, export, remove). In Figure 21 the user interface view of the Business Analytics is shown. More precisely, Pentaho Data Integration delivers a graphical design environment for ETL operations of the input stream data. In addition, a variety of dashboards (e.g., on the right) can be configured combining data source via QlikView.

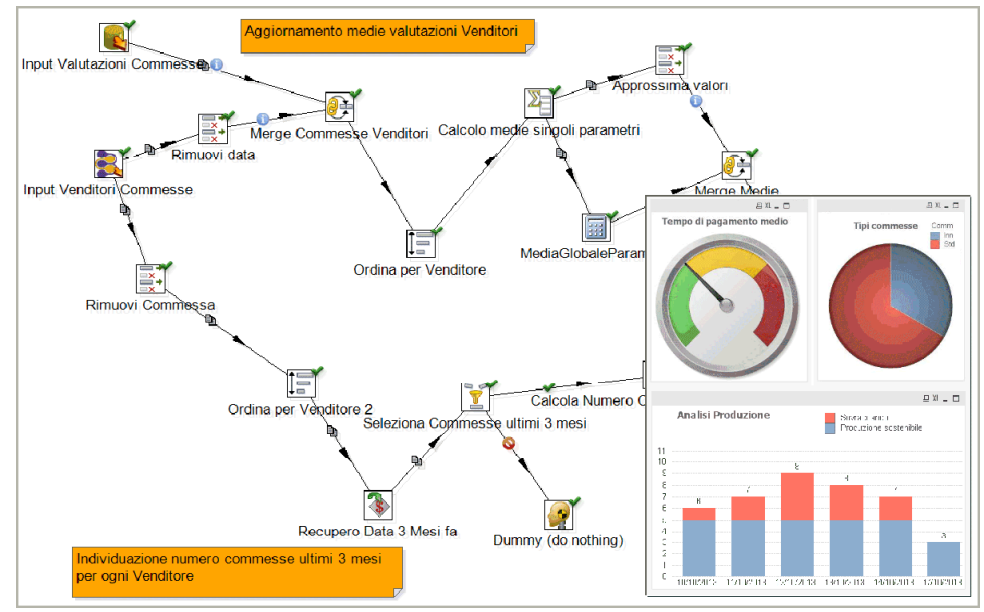

Figure 21. User interface view of the Business Analytics, created via Pentaho Data Integration and QlikView.

\subsection{System experimentation}

Since the system has been developed via integration and customization of a number of open source software products, a two-level test has been carried out. marized in Table 4. This kind of test has been managed by one software company participating to the project, and 4 companies involved in business collaborations. Each use case has been carried out either 2 times (whenever no fault is discovered) or 4 times (whenever some faults are discovered). More specifically: (a.1) each test case is tested by the software company, via an independent test team for internal acceptance and for creating the user's guides; (a.2) in each participating company a staff responsible for related test cases is designated; such staff is then trained by the software company; each test case is then tested by the staff; (a.3) in case of faults, the test team of the software company is in charge of carrying out again the test case with the new software release; (a.4) the test case is performed again by the participating company with the new 
Table 4 Unit test: number of test cases for each component.

\begin{tabular}{lr}
\hline \multicolumn{1}{c}{ Component } & \multicolumn{1}{c}{$\begin{array}{c}\text { No. of } \\
\text { test cases }\end{array}$} \\
\hline Enterprise Service Portal & 27 \\
Workflow engine and modeler & 11 \\
Semantic engine and modeler & 9 \\
Multiagent System Manager & 8 \\
Business Analytics & 16 \\
\hline
\end{tabular}

\subsubsection{System test}

It comprises the execution of 5 real-world order planning instances, summarized in Table 5 as end-to-end scenarios, to verify that the integrated system meets the business requirements. More precisely, 9 companies have been directly involved in the integration test: 4 companies who are partners of the project, and 5 client companies. Further companies have been indirectly involved as sub-contractor or supplier companies. The partners roles are: mechanical firm, electrical firm, assembling firm (who is also front-end responsible for the product sale), sub-contractor, and supplier.

Table 5 System test: business scenarios and related features.

\begin{tabular}{|c|c|c|}
\hline Business Scenario & Description & Features \\
\hline $\begin{array}{l}\text { a) Anti-vibration } \\
\text { component }\end{array}$ & $\begin{array}{l}\text { A system used to attenuate vibration on } \\
\text { vehicles }\end{array}$ & $\begin{array}{l}\text { Type of order: standard } \\
\text { Partners involved: } 3 \\
\text { External subcontractors: yes } \\
\text { Business documents: } 20\end{array}$ \\
\hline b) Painting machine & $\begin{array}{l}\text { A machine designed to support process } \\
\text { chains }\end{array}$ & $\begin{array}{l}\text { Type of order: innovative } \\
\text { Partners involved: } 3 \\
\text { External subcontractors: yes } \\
\text { Business documents: } 11\end{array}$ \\
\hline c) Mors component & $\begin{array}{l}\text { A system for disc manufacturing via } \\
\text { compression. }\end{array}$ & $\begin{array}{l}\text { Type of order: standard } \\
\text { Partners involved: } 2 \\
\text { External subcontractors: no } \\
\text { Business documents: } 9\end{array}$ \\
\hline d) Slab press & $\begin{array}{l}\text { A machine for leather ironing and } \\
\text { embossing }\end{array}$ & $\begin{array}{l}\text { Type of order: innovative } \\
\text { Partners involved: } 2 \\
\text { External subcontractors: yes } \\
\text { Business documents: } 15\end{array}$ \\
\hline e) Wooden Drum & A machine in Iroko wood for tanning & $\begin{array}{l}\text { Type of order: innovative } \\
\text { Partners involved: } 2 \\
\text { External subcontractors: yes } \\
\text { Business documents: } 11\end{array}$ \\
\hline
\end{tabular}


protocol, with the following main phases: (i) the client specifies the product category and its requirements; (ii) the system proposes a set of front-end companies; (iii) the client selects a frontend company and starts the agreement process on product requirements; (iv) if the order is not accepted, the client selects another front-end company; (v) if the order is accepted, the front-end company can require a set of partners for producing the components; (iv) once all partners have been selected, the front-end company can send the budget to the client; (v) if the budget is accepted the process ends; (vi) if the budget is not accepted the client can select another front-end company.

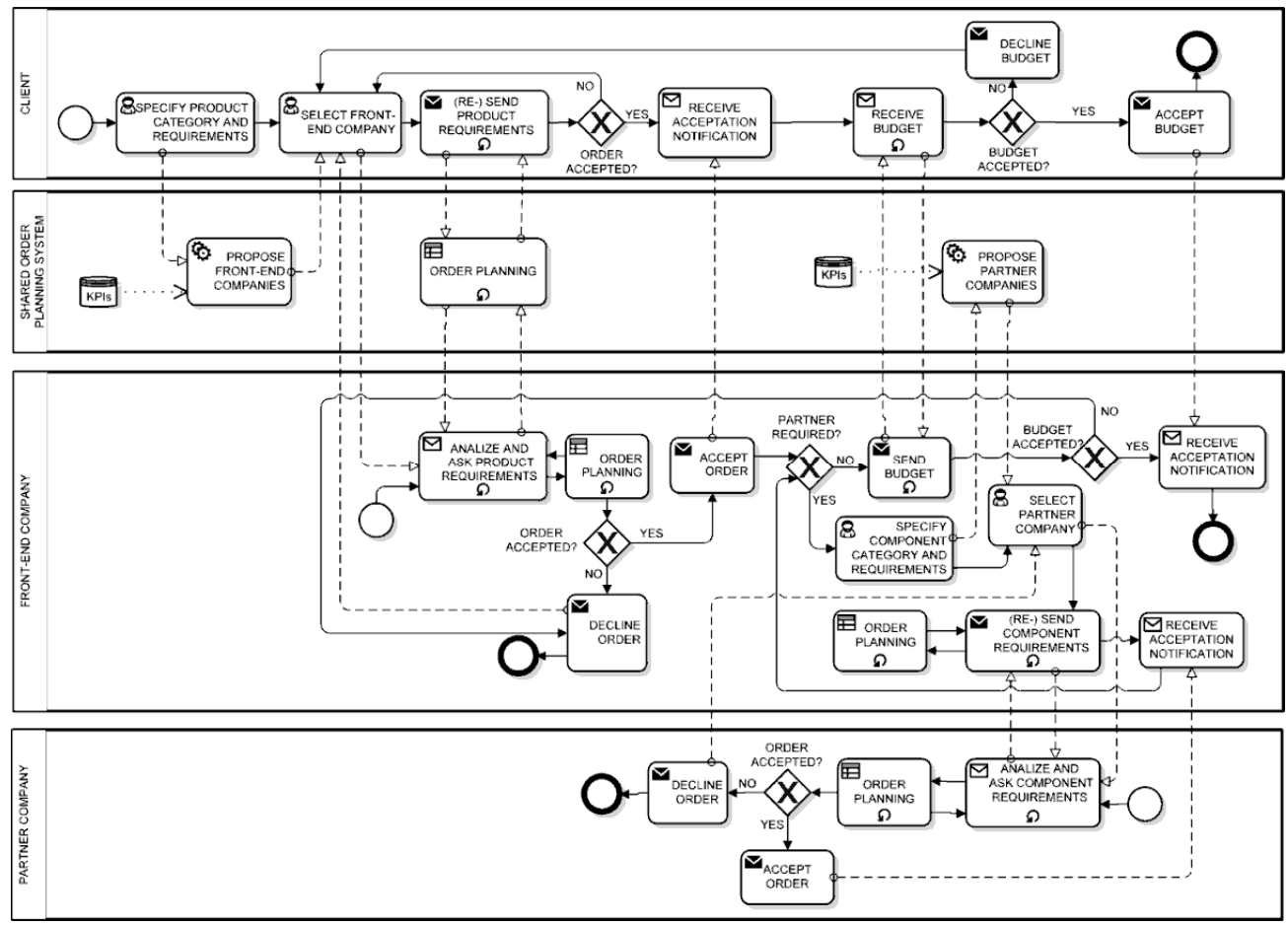

Figure 22. The main phases of the protocol for the collaborative planning of orders in the pilot scenario.

The collaboration protocol was modeled involving the partner companies, and using the methodology of Sharp (2009). It comprises business rules and collaborative analytics, for distributed decision support and data aggregation, respectively. More precisely, in Figure 22 the business rule tasks "order planning" have been developed on the basis of the business logic 990 (CSFs), based on the business rules.

991 Table 6 CSFs and KPIs based on the business rules of Figure 5 and Figure 6.

\begin{tabular}{lll}
\hline Company & CSF & KPI \\
\hline $\begin{array}{l}\text { Mechanical } \\
\text { firm }\end{array}$ & $\begin{array}{c}\text { (i) to better exploit the production capacity } \\
\text { for the standpoint of innovation }\end{array}$ & (i) percentage of innovative orders \\
& $\begin{array}{l}\text { (ii) to improve the exploitation of the } \\
\text { Electrical }\end{array}$ & (ii) average exploitation and saturation of \\
firm & production capacity in general & the production capacity \\
\hline
\end{tabular}


(iii) to speed up payment time

(iii) average payment time

$\begin{array}{lll}\text { Overall (iv) to improve the capacity to follow the } & \text { (iv) percentage of orders revised by the } \\ \text { Community } & \text { client's demand } & \text { client }\end{array}$

993

The service tasks "propose front-end companies" and "propose partner companies", feed by the data storage "KPIs", have been developed with the technique presented in Sections 4.4 and 5, and a seller/buyer rating. The rating is based on KPIs which are provided as a 1-to-5 relational feedback at the end of the collaboration, and summarized in Table 7.

998

Table 7 KPIs related to the seller/buyer rating.

999

\begin{tabular}{lll}
\hline $\begin{array}{l}\text { Company } \\
\text { Type }\end{array}$ & KPI name & KPI description \\
\hline Seller & (i) Adequacy & (i) the price is adequate to its yielded profit \\
& (ii) Reliability & (ii) the condition/level of the item/service matches its requirements \\
& (iii) Customization & (iii) personalized requirements can be implemented \\
& (iv) Expected delivery time & (iv) frequency and impact of delays \\
& (v) Post-sale service & (v) availability to damage repair and protection \\
& (vi) Communication & (vi) satisfied with the seller's communication \\
\hline Buyer & (i) Payment & (i) payment deadlines observed \\
& (ii) Changes & (ii) frequent running changes \\
& (iii) Communication & (iii) availability to interaction and meeting
\end{tabular}

1000

As an example, Fig. 23 shows a radar chart with the KPIs values that have been really

1002 associated to four seller companies. The figure is intended as a basis for the viability of analyses

1003 on the different strategies undertaken within the OCN. More specifically, it shows that the strategy

1004 of the Electrical Firm (EX), is characterized by a focus on post-sale service and expected delivery,

1005 whereas a Mechanical Firm (MY) better focuses on customization and expected delivery. In

1006 contrast, the strategic objectives of the other two Mechanical Firms ( $M X$ and $M Z)$ are oriented on

1007 adequacy and, in one case, also on post-sale service.

1008 As a result, the above business scenarios have made possible the initial roll-out of the system

1009 into production environments. Some other pilot projects will start, in order to demonstrate that the

1010 system can achieve a certain average throughput in terms of CSFs, by improving the innovative

1011 production, the exploitation of the production capacity, the payment time, and the overall capacity

1012 to follow the client's demand.

1013 Currently, the project evaluation examines whether the program is successfully recruiting and

1014 retaining its intended participants, using training materials, maintaining its timelines, coordinating

1015 partners according to their collaborative processes. Once the success in functioning of the process

1016 is confirmed, subsequent program evaluation will examine the long-term impact of the program,

1017 by taking into account the quality of the outcomes.

1018

1019 


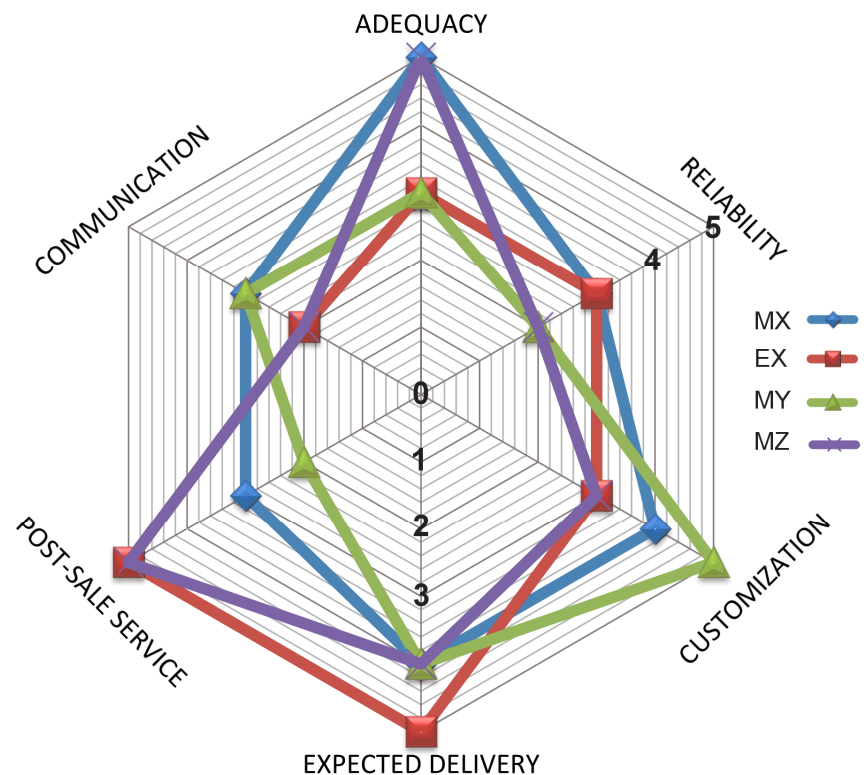

1021 Figure 23. The KPIs values associated to some seller companies.

\section{Conclusions and future works}

To model distributed business logic in OCNs is a challenging problem mainly due both to the complex interactions companies may have and the uncertainty such a dynamic environment rises. Business requirements of OCNs reveal characteristics of self-organization, distribution, transparency, and marketing concerns on data flow. A focus on OCNs business logic, supported by technological tools, leads to the integration of three technological enablers: workflow design, business rules design, and privacy-preserving collaborative analytics. First, workflow-based coordination is based on the BPMN 2 standard, and provides a fundamental technology to integrate distributed activities and data flows. Moreover, the BPMN provides a notation readily understandable by all business stakeholders, supporting the representation of the most common control-flow patterns occurring for business collaborations. Second, business rules encapsulate knowledge related to logical tasks, typically decision and control tasks. Semantic Web based on the OWL/SWRL captures all the important features needed for business rules modeling: it is a mature and well-publicized standard, with available training materials, conformant technology implementations. Semantic Web documents are very flexible; they can be joined and shared, allowing many different arrangements of rule bases. Groups of rules and facts can be easily used with distributed strategies. Third, marker-based stigmergy allows protecting business privacy and enabling self-aggregation, thus supporting collaborative analytics when combined with workflows. The above enablers have been discussed and experimented with real-world data, through a pilot scenario of collaborative order planning. A suitable architectural model is also presented, together with specific software tools implementing the most important modules.

We have designed and implemented the DLIWORP approach under the research and innovation project entitled "PMI 3.0", which has been co-financed by the Tuscany Region (Italy) for the growth of the small-medium enterprises. The approach was first implemented on a 
technical proof of concept, which demonstrated the feasibility of the ideas, verifying that the

1048 presented concepts have the potential of being used, and establishing that the system satisfies the

1049 fundamental aspects of the purpose it was designed for, by touching all of the technologies in the

1050 solution. This first prototype was used as a demonstrator to prospective companies. Subsequently

1051 the prototype was engineered by a software company, who determined the solution to some

1052 technical problems (such as how the different companies' systems might technically integrate) and

1053 demonstrated that a given configuration can achieve a certain throughput. Some pilot projects have

1054 already been started for an initial roll-out of the system into production environments. As a future

1055 work, the system will be cross-validated on different real-world scenarios, involving companies of

1056 different sizes and markets, in order to be consolidated as a design methodology. Thus, the

1057 validation of the proposed ideas has been so far partially achieved. Indeed, a concrete business

1058 infrastructure was successfully implemented, and it was possible to create given instances of the

1059 processes. However, the approach can be exhaustively tested with many scenarios and many real

1060 business situations.

\section{Acknowledgements}

1062 This research has been partially supported in the research and innovation project entitled "PMI 1063 3.0", which has been co-financed by the Tuscany Region (Italy) for the growth of the small1064 medium enterprises.

\section{References}

1066

Afsarmanesh, H., Camarinha-Matos, L. M., and Msanjila, S. S. (2010). Models, Methodologies, and Tools Supporting Establishment and Management of Second-Generation OCNs. IEEE Transactions on Systems, Man, and Cybernetcs - Part C, 41(5), 692-710.

Andrés, B., and Poler, R. (2013). Relevant problems in collaborative processes of non-hierarchical manufacturing networks. Journal of Industrial Engineering and Management, 6(3): 723-731.

Andrés, B., and Poler, R. (2014). Research on collaborative processes in non-hierarchical manufacturing networks. Technological Innovation for Collective Awareness Systems. IFIP Advances in Information and Communication Technology, 423: 21-28.

Appio, F.P., Martini, A., and Gastaldi, L. (2016). Perspectives on inter-organizational and collaborative innovation. International Journal of Technology Management, forthcoming.

Avvenuti, M., Cesarini, D., and Cimino, M.G.C.A. (2013). MARS, a multi-agent system for assessing rowers' coordination via motion-based stigmergy. Sensors, 13(9): 12218-12243.

Baldwin, C., and Von Hippel, E. (2011). Modeling a Paradigm Shift: From Producer Innovation to User and Open Collaborative Innovation. Organization Science, 22(6): 1399-1417.

Barut, M., Faisst, W., and Kanet, J.J. (2002). Measuring Supply Chain Coupling: An Information System Perspective. European Journal of Purchasing and Supply Management, 8(3): 161-171.

Bates, B. J. (1989). Information as an economic good: A reevaluation of theoretical approaches. In B. D. Ruben and L. A. Lievrouw. Mediation, Information, and Communication. New Brunswick, NJ: Transaction Publishers. 
Bechini, A., Cimino, M.G.C.A., Marcelloni, F., Tomasi, A. (2008) Patterns and technologies for enabling supply chain traceability through collaborative e-business. Information and Software Technology, 50(4): 342-359.

Bonabeau, E., Theraulaz, G., Deneubourg, J.-L., and Camazine, S. (1997). Self-organisation in social insects. Trends in Ecology and Evolution, 12(5), 188-193.

Bonabeau, E., Dorigo, M., Theraulaz, G. (1999). Swarm intelligence: From natural to artificial systems. Oxford University Press, New York.

Brambilla, M., Fraternali, P. and Vaca, C. (2011a). A Notation for Supporting Social Business Process Modeling. In Dijkman, R., Hofstetter, J., and Koheler, J. (eds.) Business Process Model and Notation, Lecture Notes in Business Information Processing 2011, 95: 88-102.

Brambilla, M., Fraternali, P., and Vaca, C. (2011b). BPMN and Design Patterns for Engineering Social BPM Solutions. 4th Workshop on Business Process Management and Social Software (BPMS2'11), co-located with BPM 2011, August 2011, Clermont-Ferrand, France.

Bullinger, A.C. (2008). Innovation and Ontologies: Structuring the Early Stages of Innovation Management. Dissertation Technische Universität München, Gabler.

Camarinha-Matos, L.M. and Afsarmanesh, H. (2007). A framework for virtual organization creation in a breeding environment. Annual Reviews in Control, 31(1): 119-135.

Camarinha-Matos, L.M., Afsarmanesh, H., Galeano, N., and Molina, A. (2009). Collaborative networked organizations - Concepts and practice in manufacturing enterprises. Computers \& Industrial Engineering, 57(1): 46-60.

Camarinha-Matos, L.M. (2013). Collaborative networks: A mechanism for enterprise agility and resilience. In: Enterprise Interoperability VI - Interoperability for Agility, Resilience, and Plasticity of Collaborations, K. Mertins et al. (eds.). Springer.

Camarinha-Matos, Luis M., and Hamideh Afsarmanesh, eds (2014). Collaborative Systems for Smart Networked Environments: Proceedings of the 15th IFIP WG 5.5 Working Conference on Virtual Enterprises, PRO-VE 2014, Amsterdam, The Netherlands, October 6-8, 2014, Vol. 434. Springer.

Carbone, F., Contreras, J., Hernández, J.Z., and Gomez-Perez, J.M. (2012). Open Innovation in an Enterprise 3.0 framework: Three case studies. Expert Systems with Applications, 39(10): 89298939.

Chen, T.Y. (2008). Knowledge sharing in virtual enterprises via an ontology-based access control approach. Computers in Industry, 59(5): 502-519.

Chen, Y., Kreulen, M., Campbell, C., and Abrams, C. (2011). Analytics ecosystem transformation: a force for business model innovation. Proceedings of the 2011 Annual SRII Global Conference, IEEE Computer Society, Washington, 11-20.

Chen, H., Chiang, R. H., and Storey, V. C. (2012). Business Intelligence and Analytics: From Big Data to Big Impact. MIS Quarterly, 36(4): 1165-1188.

Chesbrough, H.W. (2003). Open Innovation: The New Imperative for Creating and Profiting from Technology. Boston: Harvard Business School Press.

Chesbrough, H.W., and Crowther, A.K. (2006). Beyond high tech: early adopters of open innovation in other industries. $R \& D$ Management, 36(3): 229-236.Chesbrough, H.W., and 
Schwartz, K. (2007). Innovating Business Models with Co-Development Partnerships. Research-Technology Management, 50(1): 55-59.

Cimino, M.G.C.A., and Marcelloni, F. (2011). Autonomic tracing of production processes with mobile and agent-based computing. Information Sciences, 181(5): 935-953.

Clauss, T., and Spieth, P. (2015). Governance of open innovation networks with national vs. international scope. Proceedings of the $25^{\text {th }}$ ISPIM Conference, Budapest, Hunagry.

Curley, M., and Formica, P. (2013). The Experimental Nature of New Venture Creation, Capitalizing on Open Innovation 2.0. Springer.

Curley, M., and Salmelin, B. (2013). Open Innovation 2.0: A New Paradigm. White Paper. Available online: http://ec.europa.eu/information_society/newsroom/cf/dae/document.cfm? doc_id=2182.

Das, T.K., and Teng, B.S. (1998). Between trust and control: developing confidence in partner cooperation in alliances. Academy of Management Review, 23(3): 491-512.

Davenport, T. H., and Harris, J. G. (2007). Competing on Analytics. New York (NY): Harvard Business School Press.

Davenport, T. H., Harris, J. G., and Morrison, R. (2010). Analytics at Work: Smarter Decisions, Better Results. New York (NY): Harvard business Review Press.

Deeds, D.L., and Hill, C.W.L. (1996). Strategic alliances and the rate of new product development: an empirical study of entrepreneurial biotechnology firms. Journal of Business Venturing, 11(1): 41-55.

Dhakal, P. (2009). The law of rule: centralized, decentralized and distributed systems. Report for CFFN, NRN-Canada, NRNA.

Durugbo, C. (2015). Modelling information for collaborative networks. Forthcoming on Production Planning \& Control.

European Commission (2013). Open Innovation 2013. Available online: http://www.oinet.eu/attachments/article/73/OpenInnovationYearbook2013.pdf.

Escribano, A., Fosfuri, A. and Tribò, J.A. (2009). Managing external knowledge flows: the moderating role of absorptive capacity. Research Policy, 38(1): 96-105.

Eriksson, H. E., and Penker, M. (1999). Business modeling with UML: Business Patterns at Work. John Wiley \& Sons.

Ermilova, E., and Afsarmanesh, H. (2006). Competency and profiling management in virtual organization breeding environments. In L.M. Camarinha-Matos, H. Afsarmanesh and M. Ollus, eds. Network-centric collaboration and supporting frameworks. New York: Springer, 131-142.

Fabrizio, K.R. (2009). Absorptive capacity and the search for innovation. Research Policy, 38(2): 255-267.

Fagerberg, J. (2005). Innovation: a guide to the literature. In Fagerberg, J.,Mowery, D., Nelson, R. (Eds.), The Oxford Handbook of Innovation. Oxford University Press, Oxford.

Fiala, P. (2005). Information Sharing in Supply Chains. Omega, 33(5): 419-423.

Fraternali, P., Brambilla, M., and Vaca, C. (2011). A model-driven approach to social BPM applications. Social BPM. Future Strategies Inc.(May 2011). 
Furno, A., Zimeo, E. (2014). Context-aware Composition of Semantic Web Services. Mobile Networks and Applications, 19:235-248.

Gastaldi, L., Appio, F.P., Martini, A., and Corso, M. (2015). Academics as orchestrators of continuous innovation ecosystems: Towards a fourth generation CI initiatives. International Journal of Technology Management, 68(1/2): 1-20.

Gloor, P. (2006). Swarm Creativity, Competitive Advantage Through Collaborative Innovation Networks. Oxford University Press.

Grefen, P., Mehandjiev, N., Kouvas, G., Weichhart, G., Eshuis, R. (2009). Dynamic business network process management in instant virtual enterprises. Computers in Industry 60:86-103.

Heidenreich, S., Landsperger, J., and Spieth, P. (2014). Are innovation networks in need of a conductor? Examining the contribution of network managers in low and high complexity settings. In press on Long Range Planning.

Holland, O., and Melhuish, C. (1999). Stigmergy, self-organization, and sorting in collective robotics. Artificial Life, 5(2): 173-202.

Iansiti, M., and Levien, R. (2004). Strategy as Ecology. Harvard Business Review, March.

Jelasity, M., Babaoglu, O., and Laddaga, R. (2006). Guest Editors' Introduction: Self-Management through Self-Organization. Intelligent Systems IEEE, 21(2): 8-9.

Jung, J. J. (2011). Boosting social collaborations based on contextual synchronization: An empirical study. Expert Systems with Applications, 38(5), 4809-4815.

Katila, R. and Ahuja, G. (2002). Something old, something new: a longitudinal study of search behavior and new product introduction. Academy of Management Journal, 45(6): 1183-1194.

Kiemen, M. (2011). Self-organization in Open Source to support collaboration for innovation. Proceedings of the XXII ISPIM Conference held in 12-15 June 2011, Hamburg, Germany.

Krovi, R., Chandra, A., and Rajagopalan, B. (2003). Information Flow Parameters for Managing Organizational Processes. Communications of the ACM, 46 (2): 77-82.

Leymann, F., and Roller, D. (2000). Production Workflow: Concepts and Techniques. Prentice Hall.

Levine, S.S., and Prietula, M. (2013). Open Collaboration for Innovation: Principles and Performance. Organization Science, 25(5): 1414-1433.

Li, G. and Wei, M. (2014). Everything-as-a-service platform for on-demand virtual enterprises, Information Systems Frontiers, 16(3):435-452.

Liu, C., Li, Q., and Zhao, X. (2009). Challenges and opportunities in collaborative business process management: Overview of recent advances and introduction to the special issue, Information Systems Frontiers, 11(3): 201-209.

Loss L., and Crave S. (2011). Agile Business Models: an approach to support collaborative networks. Production Planning \& Control, 22(5-6): 571-580.

Macedo, P., and Camarinha-Matos, L.M. (2013). A qualitative approach to assess the alignment of Value Systems in collaborative enterprises networks. Computers \& Industrial Engineering, 64(1): 412-424.

Macedo, P., Cardoso, T., Camarinha-Matos, L.M. (2013). Value Systems Alignment in Product Servicing Networks, Collaborative Systems for Reindustrialization, IFIP Series, 408:71-80. 
Msanjila, S.S., and Afsarmanesh, H. (2006). Assessment and creation of trust in OCNs. In Camarinha-Matos, L.M., Afsarmanesh, H., and Ollus, M. (eds.), Network-centric collaboration and supporting frameworks, Springer.

Msanjijla, S.S., and Afsarmanesh, H. (2011). On modelling evolution of trust in organisations towards mediating collaboration. Production Planning \& Control, 22(5-6): 518-537.

Meech, A. (2010). Business Rules Using OWL and SWRL. Advanced in Semantic Computing, 2: 23-31.

Moore, J.F. (1996). Death of competition: leadership and strategy in the age of business ecosystems. John Wiley \& Sons.

Msanjila, S.S. and Afsarmanesh, H. (2011). On modelling evolution of trust in organizations towards mediating collaboration. Production Planning \& Control, 22(5-6): 518-537.

OMG (Object Management Group). (2011) Business Process Model and Notation (BPMN), Version 2.0, Official specification, January 2011. Available online: http://www.omg.org/spec/ BPMN/2.0.

Ollus, M., Jansson, K., Karvonen, I., Uoti, M., and Riikonen, H. (2011). Supporting collaborative project management. Production Planning \& Control, 22(5-6): 538-553.

Palley, A.B., and Kremer, M. (2014). Sequential Search and Learning from Rank Feedback: Theory and Experimental Evidence. Management Science, 60(10): 2525-2542.

Panchal, J.H. (2010). Coordination in collective product innovation. Proceedings of the ASME 2010 International Mechanical Engineering Congress \& Exposition IMECE 2010 November 12 - 18, 2010, Vancouver, BC, Canada.

Patnayakuni, R., Rai, A. and Seth, N. (2006). Relational Antecedents of Information Flow Integration for Supply Chain Coordination. Journal of Management Information Systems, 23(1): 13-49.

Peñaranda Verdeza, N., Galeano, N., Romero, D., Mejia, R., Molina, A. (2009). Collaborative Engineering Environments for Virtual Organisations. International Journal of Information Technology and Management, 8(3):298-320.

Picard, W. (2006). Support for adaptive collaboration in Professional Virtual Communities based on negotiations of social protocols. International Journal of Information Technology and Management, 8(3):298-320.

Picard, W., Paszkiewicz, Z., Gabryszak, P., Krysztofiak, K., and Cellary, W. (2010). Breeding virtual organizations in a service-oriented architecture environment. SOA Infrastructure Tools: Concepts and Methods, 375-396.

Plisson, J., Ljubic, P., Mozetic, I., and Lavrac, N. (2007). An Ontology for Virtual Organization Breeding Environments. IEEE Transactions on Systems, Man, and Cybernetics, 37(6): 13271341.

Prahalad, C.K., and Krishnan, M.S. (2008). The new age of innovation: driving co-created value through global networks. New York: McGraw-Hill. Ramaswamy, V., and Gouillart, F. (2010). The Power of Co-Creation: Build It With Them to Boost Growth, Productivity, and Profits. Free Press. 
Puranam, P., and Vanneste, B.S. (2009). Trust and Governance: untangling a tangles web. Academy of Management Review, 34(1): 11-31.

Ray, P., and Lewis, L. (2009). Managing cooperation in e-business systems, Information Systems Frontiers, 11(2): 181-188.

Reijers, H.A., Song, M., and Jeong, B. (2009). Analysis of a collaborative workflow process with distributed actors, Information Systems Frontiers, 11(3): 307-322.

Ritala, P., Armila, L., and Blomqvist, K. (2009). Innovation orchestration capability - Defining the organizational and individual level determinants. International Journal of Innovation Management, 13(4): 569-591.

Rabelo, R. J., Gusmeroli, S., Arana, C., \& Nagellen, T. (2006). The ECOLEAD ICT infrastructure for collaborative networked organizations. In L. M. Camarinha-Matos, H. Afsarmanesh \& M. Ollus (Eds.), Network-centric collaboration and supporting frameworks, IFIP, 224:451-460. New York, Springer.

Rabelo, R. J., \& Gusmeroli, S. (2008). The ECOLEAD collaborative business infrastructure for networked organizations. In L. M. Camarinha-Matos \& W. Picard (Eds.), Pervasive collaborative networks, IFIP, 283:451-462. New York, Springer.

Rabelo, R. J., Costa, S., \& Romero, D. (2014). A governance reference model for virtual enterprises, collaborative systems for smart networked environments. In L. M. CamarinhaMatos \& H. Afsarmanesh (Eds.), Collaborative Systems for Smart Networked Enterprises, IFIP, 434:60-70. Berlin Heidelberg, Springer.

Romero, D., Galeano, N. and Molina, A. (2008). A virtual breeding environment reference model and its instantiation methodology. In IFIP International Federation for Information Processing, Volume 283; Pervasive Collaborative Networks; Luis M. Camarinha-Matos, Willy Picard; (Boston: Springer), pp. 15-24.

Romero, D., Galeano, N., and Molina A. (2009). Mechanisms for assessing and enhancing organisations' readiness for collaboration in collaborative networks. International Journal of Production Research, 47(17): 4691-4710.

Romero, D., and Molina, A. (2009). VO breeding environments \& virtual organizations integral business process management framework. Information Systems Frontiers , 11: 569-597.

Romero, D., and Molina, A. (2010). Virtual organisation breeding environments toolkit: reference model, management framework and instantiation methodology. Production Planning \& Control, 21(2): 181-217.

Romero, D., and Molina, A. (2011). Collaborative networked organisations and customer communities: value co-creation and co-innovation in the networking era. Production Planning \& Control, 22(5-6): 447-472.

Rothaermel, F., and Deeds, D.L. (2006). Alliance type, alliance experience and alliance management capability in high technology ventures. Journal of Business Venturing, 21(4): 429-460.

Russell, M., Still, K., Huhtamaki, J., Yu, C., and Rubens, N. (2011). Transforming Innovation Ecosystems through Shared Vision and Network Orchestration. Triple Helix IX International Conference, Stanford University, Stanford, California. 
Sharp, A., and McDermott, P. (2009). Workflow Modeling, 2nd ed.. Artech House: Boston, MA, USA.

Simões, D., Ferreira, H., and Soares, A.L. (2007). In IFIP International Federation for Information Processing, Vol. 243, Establishing the Foundation of Collaborative Networks; eds. Camarinha-Matos, L., Afsarmaresh, H., Novais, P., Analide, C. Springer. Boston, MA, USA, pp. 137-146.

Sarnikar, S. (2007). Automating knowledge flows by extending conventional information retrieval and workflow technologies. Proceedings of the 2007 Winter Conference on Business Intelligence, David Eccles School of Business, February 22 -24, UT, USA.

Steiner, A., Morel, L., and Camargo, M. (2014). Well-suited organization to open innovation: empirical evidence from an industrial deployment. Journal of Innovation Economics \& Management, 1(13): 93-113.

Sun, Y., Tan, W., Li, L., Shen, W., Bi, Z., Hu, X. (2016). A new method to identify collaborative partners in social service provider networks, Information Systems Frontiers, 18(3): 565-578.

Tangpong, C., HHUng, K.T., and Ro, Y.K. (2010). The interaction effect of relational norms and agent cooperativeness on opportunism in buyer0supplier relationships. Journal of Operations Management, 28(5): 398-414.

Ulbrick, S., Troitzsch, H., Van den Anker, F., Plüss, A., and Huber, C. (2011). How teams in netwoked organisations develope collaborative capability: processes, critical incidents and success factors. Production Planning \& Control, 22(5-6): 488-500.

Van de Vrande, V., de Jong, J.P.J., Vanhaverbeke, W., and de Rochemont, M. (2009). Open innovation in SMEs: trends, motives and management challenges. Technovation, 29(6-7): 423437.

Van der Aalst, W. M. (2009). Process-aware information systems: Lessons to be learned from process mining. In Transactions on petri nets and other models of concurrency II (pp. 1-26). Springer Berlin Heidelberg.

Velu, C., Barrett, M., Kholi, R., and Salge, T.O. (2013). Thriving in Open Innovation Ecosystems: toward a collaborative market orientation. Working Paper.

Verbeek, M. (2008). A guide to modern econometrics. Chapter 4. John Wiley \& Sons.

Wang, Y., Wang, J., and Zhang, S. (2005). Collaborative knowledge management by integrating knowledge modeling and workflow modeling. In Information Reuse and Integration, Conf, 2005. IRI-2005 IEEE International Conference on. (pp. 13-18). IEEE.

Wang, H., Peng, X., and Gu, F. (2011). The emerging knowledge governance approach within Open Innovation: its antecedents factors and interior mechanisms. International Journal of Business and Management, 6(8): 94-104.

Well, D. (2009). Collaborative Analytics - An Emerging Practice. Available Online http://www.b-eye-network.com/view/9406.

W3C (2004). SWRL: A Semantic Web Rule Language Combining OWL and RuleML. W3C Member Submission, 21 May 2004. Available online http://www.w3.org/Submission/SWRL.

W3C (2012). Web Ontology Language (OWL). November 2012. Available online http://www.w3.org/2001/sw/wiki/OWL. 
W3C (2014). Resource Description Framework (RDF). February 2014. Available online http://www.w3.org/RDF/.

1331 Zelewski, S. (2001). Ontologien - ein Ueberblick ueber betriebswirtschaftliche Anwendungsbereiche. Workshop "Forschung in schnellebiger Zeit", Appenzell.

Zeshan, F., Mohamad, R. (2011). Semantic Web Service Composition Approaches: Overview and Limitations. International Journal on New Computer Architectures and Their Applications (IJNCAA) 1(3): 640-651.

Francesco P. Appio, PhD, is Associate Professor at the Research Center (Business Group) of the École de Management Léonard de Vinci in Paris. He is member of the Regional Entrepreneurship Acceleration Program, a global initiative at MIT. Over the past two years, he has been serving as Post-doc at the University of Pisa, School of Engineering. He completed his Ph.D. in "Management" at Scuola Superiore Sant'Anna in Pisa. His main research interests deal with the antecedents (novelty and originality) and consequences (impact) of radical innovations, intellectual property, co-creation practices, and decision making tools in the fuzzy front end of innovation.

Mario G.C.A. Cimino is with the Department of Information Engineering (University of Pisa) as a Senior Researcher in Information Systems. His research focus lies in the areas of Swarm Intelligence and Business/Social Process Analysis, with particular emphasis on Stigmergic Computing, Process Mining and Simulation. He is (co-)author of more than 40 publications.

Alessandro Lazzeri is currently a Ph.D. student in Information Engineering at the University of Pisa, Italy. He received his MSc in Business Informatics from the University of Pisa in 2013. His primary research interests include multi-agent systems and swarm intelligence. He is currently working on the application of stigmergy in temporal data analysis, as a visiting Ph.D. student at the Electrical and Computer Engineering Research Facility of the University of Alberta, Canada.

Antonella Martini, $\mathrm{PhD}$, is Associate Professor at the University of Pisa where she teaches Managerial Engineering and Organization. Her main research interests involve continuous innovation and ambidexterity. She is board member of the Continuous Innovation Network (CINet) and president of CIMEA. She is author of many publications of which more than 90 at the international level.

Gigliola Vaglini is Full Professor at the University of Pisa. Her research interests include software engineering, algorithms and formal methods for concurrent and distributed system verification. She is the coordinator of the Bachelor and the two Masters in Computer Engineering of the University of Pisa, and is co-author of more than 50 articles on international journals. 